\title{
Unifying Typing and Subtyping
}

\author{
YANPENG YANG, The University of Hong Kong, Hong Kong, China \\ BRUNO C. D. S. OLIVEIRA, The University of Hong Kong, Hong Kong, China
}

\begin{abstract}
In recent years dependent types have become a hot topic in programming language research. A key reason why dependent types are interesting is that they allow unifying types and terms, which enables both additional expressiveness and economy of concepts. Unfortunately there has been much less work on dependently typed calculi for object-oriented programming. This is partly because it is widely acknowledged that the combination between dependent types and subtyping is particularly challenging.

This paper presents $\lambda I_{\leq}$, which is a dependently typed generalization of System $F_{\leq}$. The resulting calculus follows the style of Pure Type Systems, and contains a single unified syntactic sort that accounts for expressions, types and kinds. To address the challenges posed by the combination of dependent types and subtyping, $\lambda I_{\leq}$ employs a novel technique that unifies typing and subtyping. In $\lambda I_{\leq}$there is only a judgment that is akin to a typed version of subtyping. Both the typing relation, as well as type well-formedness are just special cases of the subtyping relation. The resulting calculus has a rich metatheory and enjoys of several standard and desirable properties, such as subject reduction, transitivity of subtyping, narrowing as well as standard substitution lemmas. All the metatheory of $\lambda I_{\leq}$is mechanically proved in the Coq theorem prover. Furthermore, (and as far as we are aware) $\lambda I_{\leq}$is the first dependently typed calculus that completely subsumes System $F_{\leq}$ while preserving various desirable properties.
\end{abstract}

\section{CCS Concepts: • Theory of computation $\rightarrow$ Type theory; $\bullet$ Software and its engineering $\rightarrow$ General programming languages;}

Additional Key Words and Phrases: dependent types, subtyping, object-oriented programming

\section{ACM Reference Format:}

Yanpeng Yang and Bruno C. d. S. Oliveira. 2017. Unifying Typing and Subtyping. Proc. ACM Program. Lang. 1, OOPSLA, Article 47 (October 2017), 26 pages.

https://doi.org/10.1145/3133871

\section{INTRODUCTION}

Type systems for OOP languages are becoming increasingly more expressive and complex. For example the first versions of Java were simply typed. Java 5 introduced Generics, bringing (bounded) parametric polymorphism into mainstream OOP languages. Modern OOP languages, such as Scala, go further and include several advanced features such as higher-order polymorphism [Girard 1972; Moors et al. 2008] and path-dependent types [Odersky et al. 2004; Rompf and Amin 2016]. The extra complexity of the type systems is reflected by the significant effort to develop the corresponding metatheory. A notorious example of this is the development of the foundational metatheory for Scala, which has been an ongoing effort that lasted for more than 10 years and recently culminated with the Dependent Object Types (DOT) calculus [Rompf and Amin 2016]. DOT is an impressive

Permission to make digital or hard copies of all or part of this work for personal or classroom use is granted without fee provided that copies are not made or distributed for profit or commercial advantage and that copies bear this notice and the full citation on the first page. Copyrights for components of this work owned by others than ACM must be honored. Abstracting with credit is permitted. To copy otherwise, or republish, to post on servers or to redistribute to lists, requires prior specific permission and/or a fee. Request permissions from permissions@acm.org.

(C) 2017 Association for Computing Machinery.

2475-1421/2017/10-ART47

https://doi.org/10.1145/3133871

Proc. ACM Program. Lang., Vol. 1, No. OOPSLA, Article 47. Publication date: October 2017. 
feat, which required the development of several new proof techniques to prove type-safety and other properties.

In recent years dependent types [Altenkirch et al. 2010; Augustsson 1998; Casinghino et al. 2014; Coquand and Huet 1988; Sjöberg et al. 2012; Sjöberg and Weirich 2015; Stump et al. 2008; Weirich et al. 2013] have become a hot topic in programming language research. In functional programming dependent types are now trendy. Several new functional programming languages, most notably Agda [Norell 2007] and Idris [Brady 2013], are now dependently typed. A key reason why dependent types are interesting is that they naturally lead to a unification between types and terms, which enables both additional expressiveness and economy of concepts. The added expressiveness comes from the fact that types can now depend on values. Thus it becomes possible to express types such as lists of a certain size $n$. Such sized list type ensures stronger invariants and is helpful to prevent errors such as out-of-bounds errors. The other potential benefit of dependent types, and the main motivator for our goals in this paper, is that once various different levels of syntax (such as terms and types) are unified, then redundancy of language constructs at the various levels can be avoided. This leads to an economy of concepts compared to more traditional calculi for programming languages, which have different stratified levels of syntax. In turn, the economy of concepts results in a significantly more compact metatheory, and can also lead to a reduction of the necessary implementation effort. The key enabler for unifying terms and types in dependently typed calculi is the adoption of a style similar to Pure Type Systems (PTSs) [Barendregt 1991]. In PTSs there is only a single level of syntax for terms, and types (or kinds) that are expressed using the same syntax. This is in contrast with more traditional calculi, where distinct pieces of syntax (terms, types and kinds) are separated.

Like functional languages, OOP languages can also benefit from dependent types for exactly the same reasons: added expressiveness; and economy of concepts. Given that the complexity of type systems for OOP languages is so high, techniques for bringing down such complexity, while retaining or even increasing expressiveness are certainly welcome. The economy of concepts afforded by unified syntax typical of dependently typed languages can help here, since it can significantly reduce the number of language constructs and relations needed in a calculus. Unfortunately, there has been less work on dependently typed calculi for object-oriented programming. We believe that there are essentially two primary reasons for this. The first reason, which applies to programming languages in general (not just OOP), is that the interaction between general recursion and dependent types is challenging. Essentially recursion breaks strong normalization, which many common properties in dependently typed calculi depend upon. However, this area has been actively investigated in the last few years, and a general approach [Kimmell et al. 2012; Sjöberg et al. 2012; Sjöberg and Weirich 2015; Stump et al. 2008; Yang et al. 2016], based on explicit casts for type-level computation, has emerged to provide an interesting solution for this problem. The second reason is that how to smoothly combine dependent types and subtyping is still an open problem. Subtyping is a substantial difference to traditional PTSs, which do not have such feature. The issue with subtyping is well summarized by Aspinall and Compagnoni [1996]:

\section{One thing that makes the study of these systems difficult is that with dependent types, the typing and subtyping relations become intimately tangled, which means that tested techniques of examining subtyping in isolation no longer apply.}

In essence the big difficulty is that the introduction of dependent types makes typing and subtyping depend on each other. This causes several difficulties in developing the metatheory for calculi that combine dependent types and subtyping. Practically all previous work [Aspinall and Compagnoni 1996; Castagna and Chen 2001; Chen 1997, 2003; Zwanenburg 1999] attempts to address such problem by somehow untangling typing and subtyping, which has the benefit that 
the metatheory for subtyping can be developed before the metatheory of typing. Nevertheless, several results and features prove to be challenging. For example, several systems [Aspinall and Compagnoni 1996; Zwanenburg 1999] drop the support of top types, which are essential in OOP programs to model the universal base class. Transitivity is difficult to prove as it may be entangled with other properties such as subject reduction and strong normalization. Several studies [Aspinall and Compagnoni 1996; Castagna and Chen 2001] have to use sophisticated techniques to show that transitivity holds. Pure Subtype Systems [Hutchins 2010] take a different approach, by eliminating typing and making subtyping the essential notion in the calculus. While this simplifies the syntax and typing rules, and it is a very innovative idea, the metatheory is complex. Hutchins failed to completely prove transitivity elimination and left several important lemmas that depend on transitivity, such as subject reduction as conjectures instead. Finally, it is worthwhile mentioning that we view the work on the DOT calculus as complementary to our own. The DOT calculus [Rompf and Amin 2016] has path-dependent types, which are related but different to the dependent types discussed in this paper; and it also has a very rich notion of bounds, that goes well beyond bounded quantification. However, the DOT calculus does not attempt to unify types and terms, or typing and subtyping. Also, the rich features of DOT prevent conventional properties such as transitivity elimination (although it does have type-safety and an axiomatic transitivity property).

Despite the previous work on the combination of dependent types and subtyping, no calculi has managed to subsume System $F_{\leq}$[Cardelli et al. 1994], together with its desirable properties (for example transitivity elimination and subject reduction). System $F_{\leq}$is a standard polymorphic calculus with subtyping, often identified as a canonical calculus capturing the essential OOP features (and especially bounded quantification). Given the importance of System $F_{\leq}$as a foundational model for OOP, it seems highly desirable that a dependently typed OOP calculus subsumes it.

This paper presents $\lambda I_{\leq}$, which is a dependently typed generalization of System $F_{\leq}$. To address the challenges posed by the combination of dependent types and subtyping, $\lambda I_{\leq}$employs a novel technique that unifies typing and subtyping. In $\lambda I_{\leq}$there is only one judgment that is akin to a typed version of subtyping. Both the typing relation, as well as type well-formedness are just special cases of the subtyping relation. Therefore, $\lambda I_{\leq}$takes a significantly different approach compared to previous work. Previous work essentially attempts to fight the entanglement between typing and subtyping. In contrast, what we propose with $\lambda I_{\leq}$is to embrace such tangling, and essentially collapsing the typing and subtyping relations into the same relation. This approach is different from Hutchins' technique, which simply eliminates types and typing. $\lambda I_{\leq}$retains types.

The $\lambda I_{\leq}$calculus follows the style of Pure Type Systems, and contains a single unified syntactic sort that accounts for expressions, types and kinds. It is directly based on the $\lambda I$ calculus [Yang et al. 2016], which is a dependently typed calculus with iso-types. Iso-types provide a simple form of type casts, and $\lambda I_{\leq}$adopts that idea to address the issues arising from the combination of recursion and dependent types. The novelty over $\lambda I$ is the support for OOP features such as higher-order subtyping [Pierce and Steffen 1997], bounded quantification and top types. To illustrate the expressive power of $\lambda I_{\leq}$, we show how object encodings relying on higher-order subtyping can be done in $\lambda I_{\leq}$. The resulting calculus enjoys several standard and desirable properties, such as subject reduction, transitivity of subtyping, narrowing as well as standard substitution lemmas. All the metatheory of $\lambda I_{\leq}$and has been proved in the Coq theorem prover [The Coq development team 2016]. We also provide an algorithmic version of $\lambda I_{\leq}$based on bi-directional type-checking [Pierce and Turner 2000], which is shown to be sound and complete (also proved in Coq) with respect to the declarative version. Finally we show that $\lambda I_{\leq}$completely subsumes System $F_{\leq}$in expressive power. The manual completeness proof of $\lambda I_{\leq}$over System $F_{\leq}$is presented in the extended version [Yang and Oliveira 2017]. This proof is manual due to the well-known difficulties in mechanizing completeness proofs between type systems with unified and stratified syntax [Kaiser et al. 2017]. 
In summary the contributions of this work are:

- Unified subtyping: A novel technique that unifies typing and subtyping into a single relation. This technique enables the development of expressive dependently typed calculi with subtyping.

- The $\lambda I_{\leq}$calculus: A dependently typed calculus with subtyping that uses unified syntax, and unified subtyping. The calculus supports top types, higher-order polymorphism and bounded quantification. The paper presents a declarative version of the calculus, and a sound and complete algorithmic version is discussed. A full specification of the algorithmic system is presented in the extended version of this paper [Yang and Oliveira 2017].

- Mechanized metatheory in Coq: All proofs except for the completeness theorem over System $F_{\leq}$have been mechanized and machine-checked in the Coq theorem prover ${ }^{1}$.

- Completeness of $\lambda I_{\leq}$over System $F_{\leq}$: A completeness proof showing that $\lambda I_{\leq}$subsumes System $F_{\leq}$is available in the extended version [Yang and Oliveira 2017].

- Object encodings in $\lambda I_{\leq}$: As an example illustrating the expressive power of $\lambda I_{\leq}$, we show how object encodings relying on higher-order subtyping, and originally presented in System $F_{\leq}^{\omega}\left[\right.$ Pierce and Steffen 1997] can be done in $\lambda I_{\leq}$.

\section{OVERVIEW}

In this section, we briefly introduce the concept of unified syntax and discuss the problem of combining dependent types with subtyping. We informally introduce the key features of $\lambda I_{\leq}$calculus, namely unified subtyping and the support for dependent types by explicit casts. To illustrate the suitability of $\lambda I_{\leq}$to model objects, we adapt the existential object encoding [Bruce et al. 1999; Pierce and Turner 1994] (originally based on System $F_{\leq}^{\omega}$ ) to $\lambda I_{\leq}$. The formal details of $\lambda I_{\leq}$are further discussed in Sections 3 and 4.

\subsection{Unified Syntax versus Stratified Syntax}

Pure Type Systems [Barendregt 1991] (PTSs) are a uniform framework for typed lambda calculi. PTSs feature unified syntax which defines a single syntactic category for terms, types and kinds. This brings economy in terms of syntax and defining relations over the system. In contrast, System $F_{\omega}$ [Girard 1972], a higher-order lambda calculus, is usually presented using stratified syntax [Pierce 2002], which defines terms, types and kinds in distinct syntactic categories. System $F_{\leq}^{\omega}$ [Pierce and Steffen 1997] extends System $F_{\omega}$ with subtyping and bounded quantification. Because of the separation of syntax, the subtyping relation in System $F_{\leq}^{\omega}$ needs to be defined over multiple syntactic forms of abstraction, i.e., abstraction over terms, types and type operators. This causes duplication and complexity in the metatheory.

Note that System $F_{\omega}$ (without subtyping) can also be modeled with unified syntax: it is a special case of PTSs and covered by Barendregt's $\lambda$-cube [Barendregt 1992]. It is tempting to adopt the PTS-style unified syntax in System $F_{\leq}^{\omega}$ to simplify the subtyping relation. However, there are several difficulties in applying such simplification to a higher-order system with bounded quantification. Recall that there are three different forms of abstraction in System $F_{\leq}^{\omega}$. It is hard to unify them because the abstraction can quantify over a variable using two distinct relations, i.e., typing $(x: A)$ and subtyping $(X \leq A)$ :

$\begin{array}{ll}\text { Term abstraction } & \lambda x: A . e \\ \text { Type abstraction } & \lambda X \leq A . e \\ \text { Operator abstraction } & \lambda X \leq A . B\end{array}$

${ }^{1}$ Available from https://bitbucket.org/ypyang/oopsla17. 
To obtain a uniform representation of abstraction, we need to unify the typing and subtyping relation in the first place. Moreover, calculi with PTS-style unified syntax usually allow dependent types, e.g., the calculus of constructions [Coquand and Huet 1988]. Combining dependent types and subtyping has its own problems, as discussed in the coming subsection.

\subsection{Combining Subtyping with Dependent Types}

Mutual Dependency of Typing and Subtyping. Subtyping and dependent types are well-known features of programming languages. Individually, each of them is well-studied. However, combining them in the same system is usually difficult. The major reason is that allowing dependent types makes the typing and subtyping relations entangled. The subtyping and typing ${ }^{2}$ judgments become mutually dependent. The typing judgment depends on subtyping because of the subsumption rule:

$$
\frac{\Gamma \vdash e: A \quad \Gamma \vdash A \leq B}{\Gamma \vdash e: B}
$$

Subtyping relations are defined over well-formed types, which are checked by the typing judgment in a dependently typed system. For example, the subtyping rule for the top type $(T)$, a universal supertype of any well-formed types (i.e. with kind $\star$ ), is defined as follows:

$$
\frac{\Gamma \vdash A: \star}{\Gamma \vdash A \leq \top}
$$

Circularity in the Metatheory. The mutual dependency causes circularity in the metatheory, since one cannot study properties of subtyping independently from typing. For example, $\lambda P_{\leq}$[Aspinall and Compagnoni 1996] is an extension of the second-order dependently typed calculus $\lambda P$ [Barendregt 1992] with subtyping. In $\lambda P_{\leq}$, the substitution lemmas for typing and subtyping depend on each other and require a more complicated proof by induction on four different judgments (i.e. subtyping, typing, kinding and formation) simultaneously. The transitivity of algorithmic subtyping requires types to be well-formed through beta-conversion. As a consequence, the proofs of transitivity, strong normalization and subject reduction depend on each other.

Problems of Existing Solutions. There are several existing options to deal with the circularity. One could carefully prove mutually dependent lemmas together by finding a proper decreasing metric of induction, similar to the proof of substitution lemma in $\lambda P_{\leq}$. But such method is usually too specific and cannot be generally applied to other systems, e.g., the substitution proof in $\lambda P_{\leq}$does not apply to $\lambda \Pi^{\&}$ [Castagna and Chen 2001].

Another approach is to break the mutual dependency simply by forbidding typing from occurring in the subtyping judgments. The subtyping judgments are defined over pre-terms, terms that may not be well-formed. Then one could prove results about subtyping before typing. An obvious limitation is that subtyping rules that must depend on typing are no longer supported, such as the top type rule shown above. Several systems using this method, such as PTS $\leq$ [Zwanenburg 1999], drop the support of top types because of such limitation.

\subsection{Our Solution: Unified Subtyping}

We propose a new approach to solve the circularity problem, which also simplifies the syntax. The $\lambda I_{\leq}$calculus features a single relation for both typing and subtyping, namely unified subtyping. The relation has the form:

$$
\Gamma \vdash e_{1} \leq e_{2}: A
$$

\footnotetext{
${ }^{2}$ Some stratified systems [Aspinall and Compagnoni 1996; Castagna and Chen 2001] also have the kinding judgment, which is mutually dependent on typing. We uniformly refer to them as typing.
} 
It simultaneously contains the subtyping relation, i.e., $e_{1}$ is a subtype of $e_{2}$, and the typing relation, i.e., $e_{1}$ and $e_{2}$ have type $A$. The ordinary typing judgment can be seen as a special case of unified subtyping:

$$
\Gamma \vdash e: A \triangleq \Gamma \vdash e \leq e: A
$$

We solve the circularity problem because typing and subtyping cannot be mutually dependent in the first place - they are essentially the same relation. In $\lambda I_{\leq}$, subtyping relations can be defined over well-formed terms. Subtyping rules that depend on typing are allowed without causing mutual dependencies. As a result, top types are supported in $\lambda I_{\leq}$. Moreover, the metatheory of $\lambda I_{\leq}$is significantly simplified, e.g., there is only one form of substitution lemma to be proved, as discussed in Section 4.

Bounded quantification in $\lambda I_{\leq} . \lambda I_{\leq}$adopts a unified syntax and supports bounded quantification. Because of the unified representation of typing and subtyping, instead of three separate forms of abstraction in System $F_{\leq}^{\omega}, \lambda I_{\leq}$has a single form of abstraction: $\lambda x \leq e_{1}: A . e_{2}$. By convention, the ordinary unbounded abstraction can be treated as syntactic sugar of a top-bounded one:

$$
\lambda x: A . e \triangleq \lambda x \leq \top: A . e
$$

Notice that the top type $(T)$ is generalized to have any kind $A$ instead of $\star$. With unified syntax, $\lambda I_{\leq}$has fewer language constructs than System $F_{\leq}^{\omega}$ and a simpler definition of (unified) subtyping relation (see Section 3).

\subsection{Type Casts: Dependent Types without Strong Normalization}

Most traditional dependently typed languages are strongly normalizing (i.e. all programs terminate). Strong normalization plays a fundamental role in the metatheory of those languages. However, nearly all general purpose programming languages allow non-terminating programs, so depending on strong normalization is a non-starter if we want to model traditional general purpose languages. The root of the dependency on strong normalization is the so-called conversion rule, which allows beta equality between type expressions:

$$
\frac{\Gamma \vdash e: A \quad A={ }_{\beta} B}{\Gamma \vdash e: B}
$$

The rule checks the beta-equivalence of types and encounters evaluation, which terminates if both types are strongly normalizing. Thus, the decidability of type checking relies on strong normalization. For dependently typed languages with subtyping, the conversion rule is usually subsumed by the subsumption rule (see Section 2.2), which requires the subtyping relation $\Gamma \vdash A \leq B$ to subsume beta-equivalence $A={ }_{\beta} B$. Besides decidability, the transitivity of subtyping may also depend on strong normalization if its proof requires to first normalize the types [Aspinall and Compagnoni 1996].

An alternative to the conversion rule. Recently, several existing studies [Kimmell et al. 2012; Sjöberg et al. 2012; Sjöberg and Weirich 2015; Stump et al. 2008; Yang et al. 2016] provide a way to combine general recursion with dependent types, while preserving important properties (such as decidability of type-checking). The key idea is to replace the implicit conversion rule with explicit type casts. This has the effect that term/type equality becomes weaker: two terms are only equal up to syntactic equality (not beta-equality). To recover type conversion, an explicit cast must be used. The benefit of this design is that it decouples several properties from strong-normalization.

$\lambda I_{\leq}$adopts iso-types [Yang et al. 2016], which is one of the existing approaches to type casts. Iso-types can be viewed as a generalization of iso-recursive types [Crary et al. 1999; Pierce 2002], which works for arbitrary reducible terms rather than just (type-level) fixpoints. Two cast operators, 
namely cast $\uparrow$ and cast $\downarrow_{\downarrow}$, explicitly convert the type by one-step expansion and reduction, respectively. For example, if Int is the integer type and one-step reduction $(\lambda x: \star . x)$ Int $\hookrightarrow$ Int holds, we have

$$
\begin{array}{cc}
3: \text { Int } & e:(\lambda x: \star . x) \text { Int } \\
\text { cast }_{\uparrow}[(\lambda x: \star . x) \text { Int }] 3:(\lambda x: \star . x) \text { Int } & \operatorname{cast}_{\downarrow} e: \text { Int }
\end{array}
$$

Notice that the one-step reduction relation $(\hookrightarrow)$ used in $\lambda I_{\leq}$is weak-head and call-by-name (see Section 3.2). This makes the type conversion by casts less expressive than what is provided by the implicit conversion rule. For example, one cannot convert the length-indexed vector type $\operatorname{Vec}(1+1)$ to Vec 2 by cast $\downarrow$, since the desired reduction is not at the head position.

Nevertheless, we do not consider such loss of expressiveness problematic. The absence of conversion rule significantly simplifies the metatheory of $\lambda I_{\leq}$because typing and subtyping are up to alpha-equality and strong normalization is not a necessity for proofs. Since our goal is to design a calculus for traditional programming, we do not require the ability to do full type-level computation that is required for dependently typed programming. Cast operators are still expressive enough for our purposes: to model object encodings. Furthermore, there are alternative designs of casts which use full reduction to recover the expressiveness of the conversion rules, but they introduce some extra complications to the metatheory. Alternative approaches are discussed in Section 7.

\subsection{Example: Object Encodings using $\lambda I_{\leq}$}

We show an example of object encodings in $\lambda I_{\leq}$using the existential encoding method [Bruce et al. 1999; Pierce and Turner 1994] originally based on System $F_{\leq}^{\omega}$. The encoding requires pairs, records and existential types which are not primitives but encodable in $\lambda I_{\leq}$. We first show the encoding of dependent sums which generalize pairs and existential types before discussing object encodings.

Encoding Dependent Sums. Dependent sums are pairs where the second element can depend on the first one. The dependent sum type is also called a Sigma-type: $\Sigma x: A$. $B$, where $x$ with type $A$ can occur in $B$. In $\lambda I_{\leq}$, dependent sum types can be encoded using dependent function types (i.e. Pi-types) in a similar way to Church-encoding existential types in System $F$ or $F_{\leq}$[Pierce 2002]:

$$
\begin{array}{rlr}
\Sigma x: A . B & \triangleq \Pi z: \star .(\Pi x: A . B \rightarrow z) \rightarrow z & z \text { fresh } \\
\text { pack }\left[e_{1}, e_{2}\right] \text { as } \Sigma x: A . B & \triangleq \lambda z: \star . \lambda f:(\Pi x: A . B \rightarrow z) . f e_{1} e_{2} & z \text { fresh } \\
\text { unpack } e \text { as }[x, y] \text { in } e^{\prime} & \triangleq e C\left(\lambda x: A . \lambda y: B . e^{\prime}\right) & x, y \notin \operatorname{FV}(C)
\end{array}
$$

where pack and unpack are constructor and destructor of dependent sums, respectively. $z$ is fresh such that $z \notin \mathrm{FV}\left(\Pi x: A\right.$. B). Note that $C$ is the type of $e^{\prime}$. $A$ and $B$ can be derived from the type of $e$, i.e., $\Sigma x: A$. $B$. We can show that subtyping and typing rules of dependent sums are admissible in $\lambda I_{\leq}$. The proof is trivial and available in the extended version [Yang and Oliveira 2017].

Existential types and pairs are special cases of dependent sums. Existential types specialize $A$ to kind $\star$ :

$$
\exists x . B \triangleq \Sigma x: \star . B
$$

The constructor and destructor of an existential package are simply pack and unpack operators of dependent sums, respectively. Pairs are non-dependent sums where $x$ is not free in $B$. The pair type, constructor and destructors can be encoded as follows:

$$
\begin{array}{rll}
A \times B & \triangleq \sum x: A . B & \text { where } x \notin \mathrm{FV}(B) \\
\left(e_{1}, e_{2}\right) & \triangleq \text { pack }\left[e_{1}, e_{2}\right] \text { as } \sum x: A . B & \text { where } x \notin \mathrm{FV}(B) \\
\text { fst } e \triangleq \text { unpack } e \text { as }[x, y] \text { in } x & \\
\text { snd } e \triangleq & \triangleq \text { unpack } e \text { as }[x, y] \text { in } y &
\end{array}
$$

where in the encoding of constructor, $A$ and $B$ are types of $e_{1}$ and $e_{2}$, respectively. 
Some OO concepts can be encoded in $\lambda I_{\leq}$with dependent sums, e.g., type members in Scala [Odersky et al. 2004]. Consider an abstract interface of integer sets in Scala:

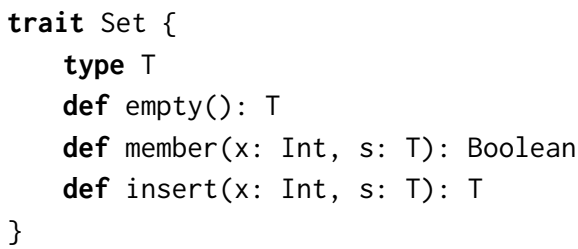

The type member $T$ represents the abstract type of a set implementation. The interface contains three methods: empty returns an empty set; member checks if an element is in the set; and insert adds an element into the set. We can use dependent sums to encode Set in $\lambda I_{\leq}$, assuming that we have primitive types (i.e. Int and Bool) and records (which can be encoded by pairs):

$$
\text { Set }=\Sigma T: \star \text {. } \text { empty }: T, \text { member }: \text { Int } \rightarrow T \rightarrow \text { Bool, insert }: \text { Int } \rightarrow T \rightarrow T\}
$$

A generic function $f$ on Set, e.g.,

$$
\operatorname{def} f(s: \text { Set })=s \cdot \operatorname{member}(3, \text { s.insert }(3, \text { s.empty }()))
$$

can be encoded as follows:

$$
f=\lambda s \text { : Set. unpack } s \text { as }[T, r] \text { in } r \text {.member } 3 \text { (r.insert } 3 \text { r.empty) }
$$

Notice that for simplicity reasons, we only encode the weak destructor of dependent sums [Schmidt 1994], i.e., the unpack operator that requires $x$ and $y$ are not free in the type $C$ of $e_{2}$. It is non-trivial to Church-encode strong dependent sums without such restriction on unpack and using only Pi-types [Cardelli 1986b]. Nevertheless, weak dependent sums are sufficient for our purpose to encode existential types and non-dependent pairs and yet more expressive than those constructs. Note that unpack operator allows unrestricted projection of existential witnesses:

$$
\lambda e:(\Sigma x: A . B) \text {. unpack } e \text { as }[x, y] \text { in } x
$$

No such operation is allowed on existential types in System $F$ or $F_{\leq}$[Amin et al. 2016].

Encoding Objects. Now that pairs and existential types can be encoded in $\lambda I_{\leq}$, we present the encoding of objects. Note again that records can be encoded with pairs using standard techniques [Pierce 2002] and that we assume $\lambda I_{\leq}$is extended with integers, pairs, records and existential types in the following text. The existential encoding of objects [Pierce and Turner 1994] is as follows:

$$
O b j=\lambda I: \star \rightarrow \star . \exists X . X \times(X \rightarrow I X)
$$

$O b j$ is a type operator, i.e., a type-level function. The binder $I$ denotes the interface. The body is an existential type which packs a pair. The pair consists of a hidden state (with type $X$ ) and methods which are functions depending on the state (with type $X \rightarrow I X$ ). For a concrete example of objects, we use the interface of cell objects [Bruce et al. 1999]:

$$
\text { Cell }=\lambda X: \star \text {. } \text { get }: \text { Int, set }: \text { Int } \rightarrow X, \text { bump }: X\}
$$

The interface indicates that a cell object consists of three methods: a getter get to return the current state, a setter set to return a new cell with a given state, and bump to return a new cell with the state increased by one.

We can define a cell object $c$ as follows:

$$
\begin{gathered}
c=\operatorname{cast}_{\uparrow}[\text { Obj Cell }] \text { pack }[\{x: \text { Int }\}, \\
\text { as CellT } \quad\{x=0\}, \lambda s:\{x: \text { Int }\} . \text { cast }_{\uparrow}[\text { Cell }\{x: \text { Int }\}] \\
\text { gget }=\text { s. } x, \text { set }=\lambda n: \text { Int. }\{x=n\}, \text { bump }=\{x=s \cdot x+1\}\})]
\end{gathered}
$$


We use the pack operator to create an existential package. The type $\{x: \operatorname{Int}\}$ corresponds to the existential binder $X$. The pair afterwards corresponds to the body of the existential type. The first component of the pair is the initial hidden state $\{x=0\}$. The second component is a function containing three methods that are defined in a record and abstracted by the state variable $s$. The definition of the three methods follows the cell object interface Cell. The result type of the package, i.e., CellT, is the one-step reduction of Obj Cell:

$$
\text { CellT }=\exists X . X \times(X \rightarrow \text { Cell } X)
$$

Note that we have two cast $\uparrow$ operators here: one over the pack operator and another over the record of methods. Due to the lack of conversion rule in $\lambda I_{\leq}$, the desired type of the object $c$ (i.e. Obj Cell) is an application, which is different from the type of the existential package (i.e. CellT). Noting that $\mathrm{Obj} \mathrm{Cell} \hookrightarrow \mathrm{CellT}$, we can use cast $\uparrow$ to do one-step type expansion for the package. Similarly, the second cast $\uparrow$ operator in the definition of methods converts the record type into Cell $\{x:$ Int $\}$. We use the following syntactic sugar for consecutive cast $\uparrow$ and pack:

$$
\text { pack }[A, e] \text { up } B \triangleq \operatorname{cast}_{\uparrow}[B]\left(\operatorname{pack}[A, e] \text { as } B^{\prime}\right)
$$

where $B \hookrightarrow B^{\prime}$, i.e., $B^{\prime}$ is the one-step reduction of $B$.

We define message passing to the object by the unpack operator to open a package. For example, sending message get to the cell object $c$ is denoted by $c \Leftarrow$ get, which is syntactic sugar of the generic message function getM:

$$
\begin{aligned}
c \Leftarrow \text { get } \triangleq & \text { getM Cell } c \\
\text { getM }= & \lambda I \leq \text { Cell }: \star \rightarrow \star . \lambda o: \text { Obj } I . \\
& \text { unpack }\left(\text { cast }_{\downarrow} o\right) \text { as }[X,(s, m)] \text { in }\left(\text { cast }_{\downarrow}(m s)\right) \text {.get }
\end{aligned}
$$

get $M$ is parameterized by interface $I$ and object $o$ with such interface, where $I$ can be any subinterface of Cell. We first use the cast $\downarrow$ operator to convert the type of $o$ from Obj I to the existential type $\exists X . X \times(X \rightarrow I X)$. Note that we extend the syntax of unpack with simple pattern matching on pairs for brevity. The hidden state is unpacked as $s$ with type $X$. The function containing methods is $m$ with type $X \rightarrow I X$. The record of methods can be obtained by applying $m$ to $s$. Noting that the subtyping relation $I X \leq$ Cell $X$ holds, the type of $m s$ can be converted from $I X$ to Cell $X$ by subsumption. Another cast $\downarrow$ further reduces Cell $X$ to record type for accessing the member get. The encoding of message bump is similar but needs to repack the resulting object:

$$
\begin{aligned}
c \Leftarrow \text { bump } \triangleq & \text { bumpM Cell } c \\
\text { bumpM }= & \lambda I \leq \text { Cell }: \star \rightarrow \star . \lambda o: \text { Obj } I . \\
& \text { unpack }\left(\text { cast }_{\downarrow} o\right) \text { as }[X,(s, m)] \text { in } \\
& \text { pack }\left[X,\left(\left(\text { cast }_{\downarrow}(m s)\right) . \text { bump }, m\right)\right] \text { up }(O b j I)
\end{aligned}
$$

since the bump method returns a record but not an object. The extra pack here is required to create a new object using the result of bump as the new hidden state.

Similarly to the original example [Bruce et al. 1999], we can examine the encoding by evaluating the expression $(c \Leftarrow$ bump $) \Leftarrow$ get using call-by-name reduction $(\hookrightarrow)$. For brevity, we omit the evaluation steps here which can be found in the extended version [Yang and Oliveira 2017]. The evaluation result is 1 as expected. We emphasize that the object encoding example exploits two fundamental features of $\lambda I_{\leq}$, namely higher-order polymorphism and explicit casts. The absence of a conversion rule does not prevent the object encoding because the required type-level computation is recovered by explicit casts. 


\section{THE $\lambda I_{\leq}$CALCULUS}

We present the $\lambda I_{\leq}$calculus in this section. The calculus features a unified syntax with only one syntactic level, and it is based on the $\lambda I$ calculus [Yang et al. 2016]. The novelty over the $\lambda I$ calculus is subtyping. To integrate subtyping, typing is unified with the subtyping relation. Thus the typing relation can be viewed as a special case of subtyping. We demonstrate the syntax, operational and static semantics of $\lambda I_{\leq}$in the rest of this section. Notice that $\lambda I_{\leq}$discussed in this section does not contain recursion, which can be supported by following $\lambda I$. We leave the discussion of recursion to Section 7.

\subsection{Syntax}

Figure 1 shows the syntax of expressions in $\lambda I_{\leq}$. It follows the unified syntax of Pure Type Systems [Barendregt 1992] where terms, types and a single kind $\star$ are defined in the same syntactic category. By convention, we still use different metavariables to indicate if expressions are terms $(e)$ or types $(A, B, C$, etc.).

Cast Operators. Cast operators cast $\uparrow$ and cast $\downarrow$ (pronounced as "cast up" and "cast down”) are used for explicit type-level computation. Types that can be converted by cast operators are also called iso-types [Yang et al. 2016]. The cast operators were introduced in the $\lambda I$ calculus as a generalization of iso-recursive types. Similarly to the fold and unfold operators in iso-recursive types [Crary et al. 1999; Pierce 2002], cast $\downarrow$ and cast $\uparrow$ convert the type of an expression by a one-step reduction or expansion, respectively. cast $\uparrow$ needs to be annotated with the result type of one-step expansion, while cast $\downarrow$ does not, since one-step reduction is deterministic (see Section 4.4).

Bounded Quantification. Functions are written as $\lambda x \leq e_{1}: A$. $e_{2}$, which support bounded quantification as in System $F_{\leq}$[Cardelli et al. 1994]. The bound term $e_{1}$ is annotated with a type $A$. Correspondingly, function types written as $\Pi x \leq e: A$. $B$ also contain a bound term $e$. Function types can be dependent if $x$ occurs free in $B$. The top type $T$ is a supertype of any well-formed term, e.g., $3 \leq T$. The top type generalizes the conventional top type in System $F_{\leq}$, which is only a supertype of well-formed types, e.g., Int $\leq \mathrm{T}$.

Syntactic Sugar. Unbounded functions $(\lambda x: A . e)$ and function types $(\Pi x: A . B)$ are not defined as primitives in the syntax. With the generalized top type, we can define them as syntactic sugar of top-bounded ones, i.e., $\lambda x \leq \top: A$. $e$ and $\Pi x \leq \top: A$. $B$ as shown in Figure 1. We also treat arrow types $A \rightarrow B$ as syntactic sugar of $\Pi x: A$. $B$ if $x$ does not occur free in $B$.

Context. The syntax of context $\Gamma$ is defined in Figure 1 . The variable binding only has the bounded form $x \leq e: A$ where the bound term $e$ has type $A$. Similar to the treatment of unbounded functions above, we can treat an unbounded variable binding as the syntactic sugar of a top-bounded binding, i.e., $\Gamma, x: A \triangleq \Gamma, x \leq \top: A$.

\subsection{Operational Semantics}

Figure 2 shows the definition of one-step reduction $(\hookrightarrow)$, which is used for both evaluation and type conversion (via cast operators). It follows the call-by-name style and is weak-head. R-BETA performs the beta reduction and does not require the argument to be a value. R-CASTELIM cancels consecutive cast $_{\downarrow}$ and cast $_{\uparrow}$. R-APP and R-CASTDN perform reduction at the head term of an application and the inner term of cast ${ }_{\downarrow}$, respectively.

Since the reduction relation $(\hookrightarrow)$ is also used for type conversion, we may encounter open terms during reduction. However, some open terms are stuck terms that are not reducible by $\hookrightarrow$. For example, an application starting with an variable: $x e_{1} e_{2} \ldots e_{n}$. Also, as the top type is generalized, 


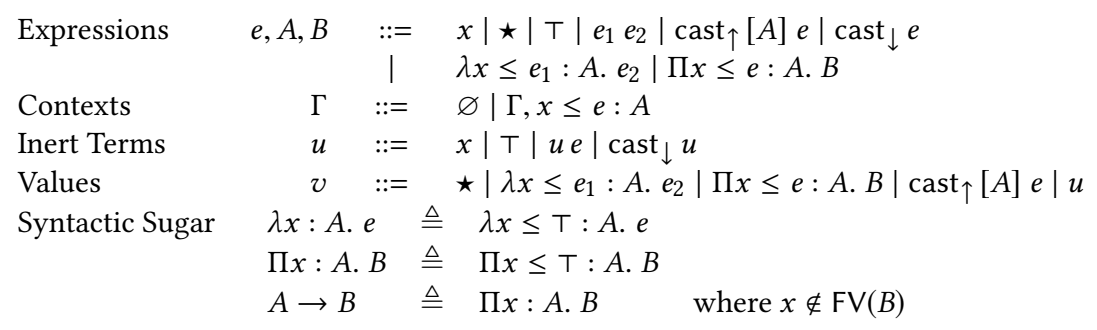

Fig. 1. Syntax

$e_{1} \hookrightarrow e_{2} \quad$ Weak-head Reduction

R-BETA

$\overline{\left(\lambda x \leq e_{3}: A . e_{1}\right) e_{2} \hookrightarrow e_{1}\left[x \mapsto e_{2}\right]}$
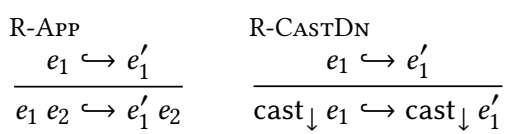

R-CAStELim

$\operatorname{cast}_{\downarrow}\left(\operatorname{cast}_{\uparrow}[A] e\right) \hookrightarrow e$

Fig. 2. Operational Semantics

assuming it is a supertype of an $n$-ary function, we can have a well-formed but stuck term such as $\top e_{1} e_{2} \ldots e_{n}$. Furthermore, if we replace $x$ and T in both stuck terms by cast ${ }_{\downarrow} x$ and cast $\downarrow$ $\top$ respectively, they still cannot be reduced.

We introduce a syntactic category called inert terms to cover such stuck terms. The terminology is inspired by the fireball calculus [Accattoli and Guerrieri 2016; Paolini and Della Rocca 1999]. Figure 1 shows the definition of inert terms, ranged over by metavariable $u$. Two base inert terms are variables and the top type. Compound inert terms are either an application leading with an inert term, i.e., $u$ e, or down-cast inert term, i.e., cast $\downarrow u$. We treat inert terms as values. Figure 1 shows the syntax of values, ranged over by metavariable $v$, as shown in Figure 1. A value can either be the kind $\star$, a function, a function type, a cast $\uparrow$ term or an inert term.

There are several alternative designs on reduction rules and syntax of values, e.g., beta-top $(\beta T)$ reduction [Pierce and Steffen 1997] and cast $\uparrow[A] v$ as a value [Pierce 2002; Yang et al. 2016]. We will discuss these designs and their trade-offs later in Section 7.

\subsection{Static Semantics}

Figure 3 shows the rules of static semantics, including two judgment forms: context well-formedness $\vdash \Gamma$ and unified subtyping $\Gamma \vdash e_{1} \leq e_{2}: A$. The unified subtyping judgment $\Gamma \vdash e_{1} \leq e_{2}: A$ serves as both subtyping and typing judgment. It can be interpreted as " $e_{1}$ is a subtype of $e_{2}$ and both of them have type $A$ ". The inference rules are developed to satisfy such interpretation. For brevity, if $e_{1}$ and $e_{2}$ are the same (i.e. $e_{1}=e_{2}=e$ ), we use the syntactic sugar $\Gamma \vdash e: A$ (see Figure 3 ), which also has the same form of typing judgment in traditional systems. We also use $\Gamma \vdash A: \star$ to check if type $A$ is well-formed, i.e., has the kind $\star$. Thus in $\lambda I_{\leq}$, subtyping, typing and well-formedness of types are all unified by the unified subtyping judgment:

$\begin{array}{lll} & & \Gamma \vdash e_{1} \leq e_{2}: A \\ \text { Unified Subtyping } & & \\ \text { Typing } & \Gamma \vdash e: A \triangleq \Gamma \vdash e \leq e: A \\ \text { Well-formed Types } & \Gamma \vdash A: \star \triangleq \Gamma \vdash A \leq A: \star\end{array}$


$\vdash \Gamma$ Context Well-formedness

$$
\frac{\text { W-Eмpty }}{\vdash \varnothing} \quad \frac{\Gamma \vdash e: A \quad \Gamma \vdash A: \star}{\vdash \Gamma, x \leq e: A}
$$

$\Gamma \vdash e_{1} \leq e_{2}: A \quad$ Unified Subtyping

$$
\begin{aligned}
& \text { S-Ax S-VARREFL } \\
& \frac{\vdash \Gamma}{\Gamma \vdash \star \leq \star: \star} \quad \frac{\vdash \Gamma \quad x \leq e: A \in \Gamma}{\Gamma \vdash x \leq x: A} \\
& \text { S-VARTRANS } \\
& \frac{x \leq e_{1}: A \in \Gamma \quad \Gamma \vdash e_{1} \leq e_{2}: A}{\Gamma \vdash x \leq e_{2}: A} \\
& \text { S-ToP } \\
& \frac{\Gamma \vdash e: A}{\Gamma \vdash e \leq \mathrm{T}: A} \\
& \text { S-TopRefL } \\
& \frac{\Gamma \vdash A: \star}{\Gamma \vdash \mathrm{\top} \leq \mathrm{\top}: A} \\
& \Gamma \vdash e_{1}: A \quad \Gamma \vdash A: \star \\
& \frac{\Gamma \vdash e_{1} \leq e_{2}: \Pi x \leq e_{3}: B . C \quad \Gamma \vdash A \leq e_{3}: B}{\Gamma \vdash e_{1} A \leq e_{2} A: C[x \mapsto A]} \\
& \text { S-CASTUP } \\
& \frac{\Gamma \vdash B: \star \quad \Gamma \vdash e_{1} \leq e_{2}: A \quad B \hookrightarrow A}{\Gamma \vdash \operatorname{cast}_{\uparrow}[B] e_{1} \leq \operatorname{cast}_{\uparrow}[B] e_{2}: B} \\
& \text { S-Sub } \\
& \frac{\Gamma \vdash e_{1} \leq e_{2}: A \quad \Gamma \vdash A \leq B: \star}{\Gamma \vdash e_{1} \leq e_{2}: B} \\
& \frac{\Gamma, x \leq e_{1}: A \vdash e_{2} \leq e_{2}^{\prime}: B \quad \Gamma, x \leq e_{1}: A \vdash B: \star}{\Gamma \vdash\left(\lambda x \leq e_{1}: A . e_{2}\right) \leq\left(\lambda x \leq e_{1}: A . e_{2}^{\prime}\right): \Pi x \leq e_{1}: A . B} \\
& \text { S-Prod } \\
& \Gamma \vdash A^{\prime} \leq A: \star \quad \Gamma \vdash e: A^{\prime} \quad \Gamma \vdash A: \star \\
& \frac{\Gamma, x \leq e: A \vdash B: \star \quad \Gamma, x \leq e: A^{\prime} \vdash B \leq B^{\prime}: \star}{\Gamma \vdash(\Pi x \leq e: A . B) \leq\left(\Pi x \leq e: A^{\prime} . B^{\prime}\right): \star} \\
& \text { S-CASTDN } \\
& \frac{\Gamma \vdash B: \star \quad \Gamma \vdash e_{1} \leq e_{2}: A \quad A \hookrightarrow B}{\Gamma \vdash \text { cast }_{\downarrow} e_{1} \leq \text { cast }_{\downarrow} e_{2}: B}
\end{aligned}
$$

Syntactic Sugar $\quad \Gamma \vdash e: A \triangleq \Gamma \vdash e \leq e: A$

Fig. 3. Static Semantics

A key benefit of unified subtyping is that the mutual dependency issue between typing and subtyping found in many traditional higher-order subtyping systems can be avoided since typing is just a special case of subtyping.

The context well-formedness judgment $\vdash \Gamma$ is defined inductively on the structure of $\Gamma$. Whenever adding a fresh binding $x \leq e: A$ to the context $\Gamma$, the judgment ensures $e$ has a well-formed type $A$.

We briefly introduce the basic rules and discuss the rest in detail. S-Ax defines the reflexivity of the kind $\star$ and follows the "type-in-type" axiom [Cardelli 1986b] for the typing of $\star$. S-VARREFL defines the reflexivity of a variable and its typing by looking up the context. S-VARTRANS defines the variable lookup followed by transitivity, which follows the algorithmic version of System $F_{\leq}$[Curien and Ghelli 1992].

Generalized Top Type. S-Top defines subtyping for the generalized top type: a supertype of any term $e$ which has the same type $A$ as $e$. A special case is when $e$ is also a top type. For this case we need to define the reflexivity of top type as in the rule S-TopRefL, which indicates that the top type can have any well-formed type $A$. In other words, any well-formed type can be inhabited by the generalized top type, which causes logical inconsistency. Note that allowing "type-in-type" axiom in S-Ax already brings logical inconsistency [Barendregt 1992]. Our goal is to investigate the calculus for traditional programming that allows general recursion, which is logically inconsistent any way. Thus, we do not consider generalized top type or "type-in-type" axiom problematic. With top type generalized, bounded and unbounded quantification are unified, which significantly simplifies the system. 
Functions and Function Types. S-ABs defines the relation between functions, which follows the invariant rule for type operators in System $F_{\leq}^{\omega}$ [Pierce and Steffen 1997]. It requires the bounds and argument types being compared to be identical. The first line of premises in S-ABs checks the well-formedness of binding. The second line of premises checks if the function bodies are covariant and their type is well-formed.

S-ProD defines the relation between function types. Unlike S-ABS, it only requires the bounds to be identical. The argument types can vary and are contravariant. Such design follows the Kernel Fun variant [Cardelli and Wegner 1985] of System $F_{\leq}$. S-Prod can be viewed as a combination of the subtyping rules for arrow types and universal types of System $F_{\leq}$:

$$
\begin{array}{ll}
\begin{array}{l}
\text { FS-ARrow } \\
\Delta \vdash T_{1} \leq U_{1} \quad \Delta \vdash U_{2} \leq T_{2}
\end{array} & \begin{array}{c}
\text { FS-ForALL } \\
\Delta \vdash U_{1} \rightarrow U_{2} \leq T_{1} \rightarrow T_{2}
\end{array} \\
\Delta \vdash \forall X \leq U . T_{1} \leq \forall X \leq U . T_{2}
\end{array}
$$

The first premise of S-PROD checks the contravariance of argument types, similar to the rule for arrow types. The last premise checks the covariance of co-domains of function types with bound fixed, similar to the rule for universal types. Other premises check the well-formedness.

Pointwise Subtyping. S-App defines subtyping between applications and uses a pointwise subtyping rule originated from System $F_{\leq}^{\omega}$ [Pierce and Steffen 1997], which is also used in many systems with higher order subtyping [Aspinall and Compagnoni 1996; Hutchins 2010; Zwanenburg 1999] When comparing two applications, we require the arguments to be identical and only compare the head terms, equivalently to type operators in $F_{\leq}^{\omega}$. The first premise of S-App ensures the head term to have a function type, e.g., $\Pi x \leq e_{3}: B$. $C$. The second premise checks the bound and typing requirements: if the argument $A$ is a subtype of $e_{3}$ and $A$ has the type $B$.

Explicit Casts and Syntactic Equality. S-CASTUp and S-CASTDN are rules for explicit cast operators. They can be seen as a generalization of typing rules of fold and unfold from iso-recursive types [Pierce 2002; Yang et al. 2016]. Weak-head reduction $(\hookrightarrow)$ is used for type-level conversion. Note that when comparing cast $\uparrow$ terms, we require the annotations to be the same. S-SuB is the subsumption rule. The second premise checks the subtyping relation between well-formed types by reusing the unified subtyping judgment. Note that S-Sub does not subsume the implicit conversion rule, which can be found in $F_{\leq}^{\omega}$ and Pure Type Systems. Because the unified subtyping judgment does not subsume beta conversion, i.e., $(\lambda x: \star . x)$ Int $\leq$ Int does not hold. As a consequence, types of expressions are equal only up to syntactic equality (i.e. alpha equality), but not beta equality Nevertheless, we can recover type-level computation through cast operators in a similar way to iso-types [Yang et al. 2016].

Algorithmic up to Subtyping. The unified subtyping rules shown in Figure 3 are declarative because of the subsumption rule S-SuB. But the system is almost algorithmic: if we ignore the typing result and only consider the subtyping part, the system becomes algorithmic. Like the algorithmic version of System $F_{\leq}$, there is no built-in transitivity rule defined in $\lambda I_{\leq}$. Actually, transitivity can be proved from other rules (see Section 4.2).

\section{THE METATHEORY OF UNIFIED SUBTYPING}

In this section, we discuss the metatheory of $\lambda I_{\leq}$by focusing on two main targets: transitivity and type safety. We emphasize here that in previous work the metatheory for the combination between dependent types and subtyping was a key difficulty, greatly due to the entanglement between the metatheory of subtyping and typing. With unified subtyping we develop a single metatheory for the new relation instead. Traditional theorems related to the metatheory of typing and subtyping can then be viewed as particular instantiations of the unified subtyping theorems. Because the 


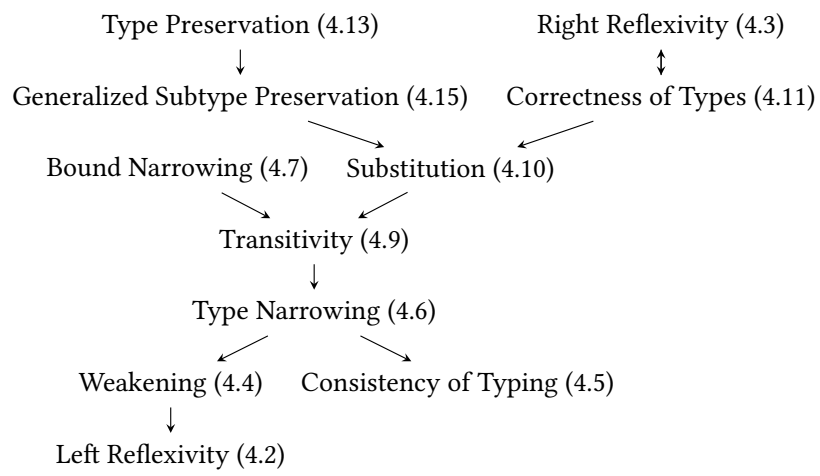

Fig. 4. Dependency of Lemmas for the Metatheory of Unified Subtyping

unified subtyping relation is new, working out the metatheory for our system actually required figuring out which theorems to prove (i.e. what form should they have); and in which order to prove them. It is crucial (and non-trivial) to prove the right theorems in the correct order. Nevertheless, once the form of the theorems and the order in which they should be proved are set, then the proofs can actually be done with simple techniques similar to those used in more traditional systems. The dependency diagram of main lemmas in this section is shown in Figure 4. We only show the proof sketch and discuss interesting cases in this section. The full proofs (mechanized in Coq) can be found in the extended version [Yang and Oliveira 2017].

\subsection{Basic Lemmas}

Before going to the proof of transitivity, we first discuss several important basic lemmas including reflexivity, weakening, consistency of typing and narrowing.

Reflexivity. The subtyping relation in System $F_{\leq}$is reflexive, i.e., $\Delta \vdash T \leq T$ holds for any well-formed type $T$ and context $\Delta$. Since unified subtyping in $\lambda I_{\leq}$tracks typing results, the relation in reflexive form, i.e., $\Gamma \vdash e \leq e: A$, works like a typing judgment $\Gamma \vdash e: A$ (recall the syntactic sugar in Figure 3). Reflexivity does not hold for arbitrary $e$ and $A$. However reflexivity does hold for any well-(sub)typed terms. That is:

Lemma 4.1 (Reflexivity). If $\Gamma \vdash e_{1} \leq e_{2}: A$, then both $\Gamma \vdash e_{1}: A$ and $\Gamma \vdash e_{2}:$ A hold.

This lemma is also called validity in some literature [Abel and Rodriguez 2008]. Here we call it "reflexivity" because conclusions are still (unified) subtyping relations in reflexive form. It also meets the interpretation of unified subtyping mentioned in Section 3.3. We separate the reflexivity lemma into two sub-lemmas by dividing the conclusion:

Lemma 4.2 (Left Reflexivity). If $\Gamma \vdash e_{1} \leq e_{2}: A$, then $\Gamma \vdash e_{1}: A$ holds.

Lemma 4.3 (Right Reflexivity). If $\Gamma \vdash e_{1} \leq e_{2}: A$, then $\Gamma \vdash e_{2}: A$ holds.

Left reflexivity can be proved by induction on the derivation of $\Gamma \vdash e_{1} \leq e_{2}: A$. However, right reflexivity is difficult to prove due to the generalized top type. Consider the case of S-Top, i.e., $\Gamma \vdash e \leq \top: A$. We know $\Gamma \vdash e: A$ from the premise. The target $\Gamma \vdash \top: A$ requires $A$ to be wellformed, i.e., $\Gamma \vdash A: \star$, as indicated by the premise of S-TopRefl. To prove $\Gamma \vdash A: \star$ from $\Gamma \vdash e: A$, we need a lemma called correctness of types (Lemma 4.11), which is not available currently. We will show the full proof later in Section 4.3. Currently without right reflexivity, we add redundant 
premises in typing rules to simplify the proofs. For example, in rule S-Prod, the third premise $\Gamma \vdash A: \star$ is derivable from the first premise $\Gamma \vdash A^{\prime} \leq A: \star$ by right reflexivity. Once right reflexivity is shown, such additional premises can be removed without changing the type system.

Weakening. The weakening lemma is standard:

Lemma 4.4 (Weakening). If $\Gamma_{1}, \Gamma_{3} \vdash e_{1} \leq e_{2}: A$ and $\vdash \Gamma_{1}, \Gamma_{2}, \Gamma_{3}$, then $\Gamma_{1}, \Gamma_{2}, \Gamma_{3} \vdash e_{1} \leq e_{2}: A$.

The proof is by induction on the derivation of $\Gamma_{1}, \Gamma_{3} \vdash e_{1} \leq e_{2}: A$. The only interesting case is when $\mathrm{S}-\mathrm{PrOD}$ is the last derivation. The last premise of S-Prod adds binding $x \leq e: A^{\prime}$ into the context $\Gamma$. We need to ensure $A^{\prime}$ is well-formed, i.e., $\Gamma \vdash A^{\prime}: \star$, as required by context well-formedness. Though not included in the premise, it can be derived by applying left reflexivity (Lemma 4.2) to the first premise, i.e., $\Gamma \vdash A^{\prime} \leq A: \star$.

Consistency of Typing. We prove a simple yet important lemma, called consistency of typing:

Lemma 4.5 (Consistency of Typing). If $\Gamma \vdash e_{1}: A$ and $\Gamma \vdash e_{1} \leq e_{2}: B$, then $\Gamma \vdash e_{1} \leq e_{2}: A$.

The proof is by induction on the derivation of $\Gamma \vdash e_{1} \leq e_{2}: B$. This lemma is the key to decoupling typing from unified subtyping. To prove $\Gamma \vdash e_{1} \leq e_{2}: A$, we can individually show 1) $e_{1}$ has the type $A$ and 2) $e_{1}$ is a subtype of $e_{2}$ regardless of typing, as long as there is some type $B$ such that $\Gamma \vdash e_{1} \leq e_{2}: B$.

Narrowing. We have two narrowing lemmas in $\lambda I_{\leq}$, type narrowing and bound narrowing:

Lemma 4.6 (Type Narrowing). Given $\Gamma_{1}, x \leq e: B, \Gamma_{2} \vdash e_{1} \leq e_{2}: C$, if $\Gamma_{1} \vdash A \leq B: \star$ and $\Gamma_{1} \vdash e: A$, then $\Gamma_{1}, x \leq e: A, \Gamma_{2} \vdash e_{1} \leq e_{2}: C$.

Lemma 4.7 (Bound Narrowing). If $\Gamma_{1}, x \leq e: B, \Gamma_{2} \vdash e_{1} \leq e_{2}: C$ and $\Gamma_{1} \vdash e^{\prime} \leq e: B$, then $\Gamma_{1}, x \leq e^{\prime}: B, \Gamma_{2} \vdash e_{1} \leq e_{2}: C$.

As indicated by the name, for a binding $x \leq e: B$ in the context, type narrowing changes its type from $B$ to a subtype $A$, while bound narrowing changes its bound from $e$ to a subtype $e^{\prime}$. We only prove type narrowing here, since bound narrowing depends on transitivity, as will be discussed later in Section 4.3. The type narrowing lemma is proved by induction on the derivation of $\Gamma_{1}, x \leq e: B, \Gamma_{2} \vdash e_{1} \leq e_{2}: C$. The only interesting case is when the last derivation uses S-VARTRANS, i.e., $e_{1}$ is a variable. It is easy to prove by the induction hypothesis when $e_{1}$ is not $x$. When $e_{1}=x$, we know $B=C$ and our target is to show $\Gamma_{1}, x \leq e: A, \Gamma_{2} \vdash x \leq e_{2}: B$. By applying the subsumption rule S-Sub and S-VArTrans, our target becomes $\Gamma_{1}, x \leq e: A, \Gamma_{2} \vdash e \leq e_{2}: A$. Note that we have $\Gamma_{1}, x \leq e: A, \Gamma_{2} \vdash e \leq e_{2}: B$ by the induction hypothesis. The only gap is the typing result, which should be $A$ but not $B$. Thus, we can apply the consistency of typing lemma (Lemma 4.5) and prove $\Gamma_{1}, x \leq e: A, \Gamma_{2} \vdash e: A$ instead, which is immediate by weakening (Lemma 4.4).

\subsection{Transitivity}

Transitivity is a desirable property of systems with subtyping. Declarative presentations of calculi often include a built-in transitivity rule:

$$
\frac{\Gamma \vdash e_{1} \leq e_{2}: A \quad \Gamma \vdash e_{2} \leq e_{3}: A}{\Gamma \vdash e_{1} \leq e_{3}: A} \text { S-TrAnS }
$$

This simplifies the proof of lemmas such as narrowing and substitution. However, noticing that $e_{1}$ and $e_{3}$ can be in any form, the rule can be applied any time during derivation, which complicates the inversion of subtyping judgments. A process called transitivity elimination [Compagnoni 1995; Pierce 2002; Pierce and Steffen 1997] can be used to avoid such complexity brought by the transitivity 
rule. The declarative system is reformulated into an algorithmic one without a transitivity rule. The transitivity property is then proved against the algorithmic system. Similarly, we formulate $\lambda I_{\leq}$without a built-in transitivity rule but only with a base case for variables (i.e. S-VARTRANS), as mentioned in Section 3.3. Next we show the proof of transitivity in $\lambda I_{\leq}$.

First, we need to generalize the form of transitivity. The form of rule S-TRANS is too restricted: conditions are required to have the same type. This causes issues when both conditions are derived from S-SuB:

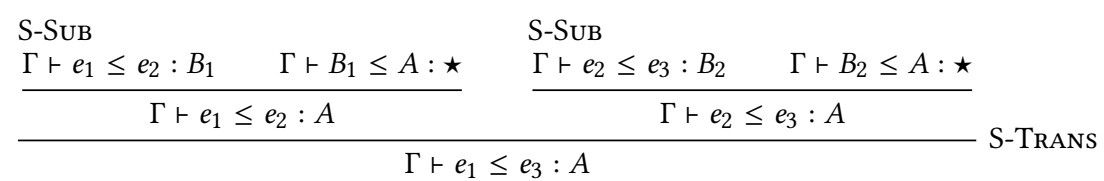

We only know $B_{1}$ and $B_{2}$ are both subtypes of $A$ but cannot determine the relation between them. The induction hypothesis cannot be applied since it requires $B_{1}$ and $B_{2}$ to be the same. Thus, we generalize the property into

$$
\frac{\Gamma \vdash e_{1} \leq e_{2}: A \quad \Gamma \vdash e_{2} \leq e_{3}: B}{\Gamma \vdash e_{1} \leq e_{3}: A} \text { S-Trans2 }
$$

where the conditions are allowed to have different types and the conclusion needs to have the same type as the first condition. The proof of the generalized transitivity is standard [Pierce 2002] by induction on the size of $e_{2}$ and an inner induction on the derivation of the first condition $\Gamma \vdash e_{1} \leq e_{2}: A$. We only discuss the interesting case when both derivations end with S-Prod. We have $e_{1}=\Pi x \leq e: A_{1} . B_{1}, e_{2}=\Pi x \leq e: A_{2} . B_{2}$, and $e_{3}=\Pi x \leq e: A_{3} . B_{3}$, with

$$
\begin{array}{lll}
\Gamma \vdash A_{2} \leq A_{1}: \star & (1) & \Gamma, x \leq e: A_{2} \vdash B_{1} \leq B_{2}: \star \\
\Gamma \vdash A_{3} \leq A_{2}: \star & (3) & \Gamma, x \leq e: A_{3} \vdash B_{2} \leq B_{3}: \star
\end{array}
$$

For clarity, we omit all derivations for well-formedness checking in the discussion, which can be trivially proved by the induction hypothesis. Our target is to prove $\Gamma \vdash A_{3} \leq A_{1}: \star$ and $\Gamma, x \leq e: A_{3} \vdash B_{1} \leq B_{3}: \star$. The first target can be obtained by combining (1) and (3) using the outer induction hypothesis since $A_{2}$ has smaller size than $e_{2}$. Noting that the context of the (2) is different from (4) and the second target, we use Lemma 4.6 to narrow the type of the binding to obtain $\Gamma, x \leq e: A_{3} \vdash B_{1} \leq B_{2}: \star$. Then we can similarly obtain the second target by the outer induction hypothesis since the size of $B_{2}$ is smaller than $e_{2}$. We conclude the generalized transitivity by the following lemma:

Lemma 4.8 (Generalized Transitivity). If $\Gamma \vdash e_{1} \leq e_{2}: A$ and $\Gamma \vdash e_{2} \leq e_{3}: B$, then $\Gamma \vdash e_{1} \leq e_{3}: A$.

Thus, the original transitivity is an immediate corollary:

Lemma 4.9 (Transitivity). If $\Gamma \vdash e_{1} \leq e_{2}: A$ and $\Gamma \vdash e_{2} \leq e_{3}: A$, then $\Gamma \vdash e_{1} \leq e_{3}: A$.

As shown in Figure 4, the proof of generalized transitivity depends on type narrowing (Lemma 4.6) and type narrowing depends on consistency of typing (Lemma 4.5). Actually, we can view consistency of typing as a special case of generalized transitivity by letting $e_{1}=e_{2}=e_{1}^{\prime}$ and $e_{3}=e_{2}^{\prime}$. This indicates that type narrowing can also be proved using generalized transitivity. Thus, an alternative approach is to prove generalized transitivity and type narrowing simultaneously. A potential issue is that this approach makes these two lemmas mutually dependent. We choose to first prove a weaker version of generalized transitivity, i.e., consistency of typing, which has a much simpler proof. Then we can show type narrowing before transitivity without causing circularity. 


\subsection{Basic Lemmas, Revisited}

Recall that in Section 4.1 we leave two lemmas unproved, i.e., right reflexivity (Lemma 4.3) and bound narrowing (Lemma 4.7), which depend on other lemmas that were not available yet. As we have proved transitivity in Section 4.2, we can recover the proof of these two lemmas.

Bound Narrowing. Similar to type narrowing (Lemma 4.6), bound narrowing (Lemma 4.7) is proved by induction on the derivation of $\Gamma_{1}, x \leq e: B, \Gamma_{2} \vdash e_{1} \leq e_{2}: C$. We consider the interesting case when the derivation ends with S-VARTrans. If $e_{1}$ is not $x$, it is trivial to prove by the induction hypothesis. If $e_{1}=x$, we have $B=C$ and our target is to show $\Gamma_{1}, x \leq e^{\prime}: B, \Gamma_{2} \vdash x \leq e_{2}: B$. By the induction hypothesis, we have $\Gamma_{1}, x \leq e^{\prime}: B, \Gamma_{2} \vdash e \leq e_{2}: B$. Noticing that by weakening (Lemma 4.4), we can obtain $\Gamma_{1}, x \leq e^{\prime}: B, \Gamma_{2} \vdash e^{\prime} \leq e: B$ from the second condition. By transitivity (Lemma 4.9), we have $\Gamma_{1}, x \leq e^{\prime}: B, \Gamma_{2} \vdash e^{\prime} \leq e_{2}: B$. Also noticing that $x \leq e^{\prime}: B \in \Gamma_{1}, x \leq e^{\prime}: B, \Gamma_{2}$, we obtain the target by the rule S-VARTrans.

Substitution. We show that the substitution lemma holds in $\lambda I_{\leq}$:

Lemma 4.10 (Substitution). If $\Gamma_{1}, x \leq e: B, \Gamma_{2} \vdash e_{1} \leq e_{2}: A$ and $\Gamma_{1} \vdash e^{\prime} \leq e: B$, then $\Gamma_{1}, \Gamma_{2}\left[x \mapsto e^{\prime}\right] \vdash e_{1}\left[x \mapsto e^{\prime}\right] \leq e_{2}\left[x \mapsto e^{\prime}\right]: A\left[x \mapsto e^{\prime}\right]$.

The proof is standard by induction on the derivation of the first condition. It is similar to the proof of bound narrowing. Transitivity and weakening are also required for the case when S-TransVAR is the last derivation. Note that the second condition $\Gamma_{1} \vdash e^{\prime} \leq e: B$ contains both subtyping requirement ( $e^{\prime}$ is a subtype of $e$ ) and typing requirement ( $e^{\prime}$ has type $B$ ). Thus, the substitution lemma in $\lambda I_{\leq}$has only one form.

Right Reflexivity. As mentioned in Section 4.1, right reflexivity (Lemma 4.3) depends on correctness of types:

Lemma 4.11 (Correctness of Types). If $\Gamma \vdash e_{1} \leq e_{2}: A$, then $\Gamma \vdash A: \star$.

But correctness of types also depends on right reflexivity. Consider the last derivation of $\Gamma \vdash e_{1} \leq$ $e_{2}: A$ is S-SuB, where the premises are $\Gamma \vdash e_{1} \leq e_{2}: B$ and $\Gamma \vdash B \leq A: \star$. The conclusion $\Gamma \vdash A: \star$ holds if we apply right reflexivity to the second premise. Thus, we prove these two lemmas simultaneously by induction on the derivation of $\Gamma \vdash e_{1} \leq e_{2}: A$. Note that the proof of correctness of types also depends on the substitution lemma (Lemma 4.10) when the derivation ends with S-App.

With both left and right reflexivity proved, we conclude the reflexivity (Lemma 4.1) holds and the interpretation of unified subtyping in Section 3.3 is correct. One key insight here is that we do not prove the full reflexivity lemma first. Otherwise, it will cause circular dependency in the metatheory (imagine merging two nodes of left and right reflexivity in Figure 4).

\subsection{Type Safety}

We prove type safety by showing type preservation and progress lemmas [Wright and Felleisen 1994]. Though both lemmas have the same form as traditional systems, the typing judgment is just syntactic sugar of unified subtyping, as mentioned in Section 3.3.

Determinacy of Reduction. We first show that the one-step reduction relation is deterministic:

Lemma 4.12 (Determinacy of Reduction). If $e \hookrightarrow e_{1}$ and $e \hookrightarrow e_{2}$, then $e_{1}=e_{2}$.

The proof is straightforward by induction on the derivation of $e \hookrightarrow e_{1}$. Note that the equality used in the conclusion is syntactic equality. The result of type-level reduction in the rule S-CASTDN (i.e. type $B$ ) is unique. Thus, the cast $\downarrow$ term is not required to be annotated with the result type. 
Type Preservation. Type preservation, also known as subject reduction [Wright and Felleisen 1994], states that reducing a term does not change its type:

Lemma 4.13 (Type Preservation). If $\Gamma \vdash e: A$ and $e \hookrightarrow e^{\prime}$, then $\Gamma \vdash e^{\prime}: A$.

However, if we try to directly prove this lemma by induction on the derivation of $\Gamma \vdash e \leq e: A$ (i.e. $\Gamma \vdash e: A$ ), the proof will get stuck. Consider the last derivation is S-CASTDN and $e \hookrightarrow e^{\prime}$ is an instance of R-CASTELIM with $e=\operatorname{cast}_{\downarrow}\left(\operatorname{cast}_{\uparrow}\left[B^{\prime}\right] e\right)$ and $e^{\prime}=e$. We have $\Gamma \vdash \operatorname{cast}_{\uparrow}\left[B^{\prime}\right] e: B$ and $B \hookrightarrow A$. By inversion of S-CAstUp, we can obtain $\Gamma \vdash e: A^{\prime}, B^{\prime} \hookrightarrow A^{\prime}$ and $\Gamma \vdash B^{\prime} \leq B: \star$. Our target is to show $\Gamma \vdash e: A$. If we can prove $\Gamma \vdash A^{\prime} \leq A: \star$, then the target can be obtained immediately by the subsumption rule S-Sub. The relation is shown as follows:

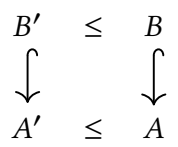

The subtyping relation in the second line requires a proof, which can be shown by the following lemma with a more general typing result other than the kind $\star$ :

Lemma 4.14 (Subtype Preservation). If $\Gamma \vdash e_{1} \leq e_{2}: A, e_{1} \hookrightarrow e_{1}^{\prime}, e_{2} \hookrightarrow e_{2}^{\prime}$, then $\Gamma \vdash e_{1}^{\prime} \leq e_{2}^{\prime}: A$.

We call this lemma subtype preservation indicating that the unified subtyping relation is preserved by reduction. Type preservation is just a special case of it when $e_{1}=e_{2}=e$ and $e_{1}^{\prime}=e_{2}^{\prime}=e^{\prime}$. A naïve proof is by induction on the derivation of $\Gamma \vdash e_{1} \leq e_{2}: A$. The substitution lemma (Lemma 4.10) is required for the case when the derivation ends with S-App and both reductions are instances of R-BETA. However, the proof gets stuck when the derivation ends with S-CASTDN, and both reductions are instances of R-CASTELIM with $e_{1}=\operatorname{cast}_{\downarrow}\left(\operatorname{cast}_{\uparrow}[B] e_{1}^{\prime}\right)$ and $e_{2}=\operatorname{cast}_{\downarrow}\left(\operatorname{cast}_{\uparrow}[B] e_{2}^{\prime}\right)$. The induction hypothesis does not work as it requires cast $\uparrow[B] e_{1}^{\prime}$ and cast $_{\uparrow}[B] e_{2}^{\prime}$ to be reducible, while both of them are values (see Figure 1). To solve this issue, we need to generalize the subtype preservation lemma into the following one:

Lemma 4.15 (Generalized Subtype Preservation). Given that $\Gamma \vdash e_{1} \leq e_{2}:$ A holds,

(1) if both $e_{1}$ and $e_{2}$ are cast $\uparrow$ terms, i.e., $e_{1}=\operatorname{cast}_{\uparrow}[B] e_{1}^{\prime}$ and $e_{2}=\operatorname{cast}_{\uparrow}[B] e_{2}^{\prime}$, and $A \hookrightarrow A^{\prime}$, $B \hookrightarrow B^{\prime}$, then $\Gamma \vdash e_{1}^{\prime} \leq e_{2}^{\prime}: A^{\prime}$;

(2) otherwise, if $e_{1} \hookrightarrow e_{1}^{\prime}$ and $e_{2} \hookrightarrow e_{2}^{\prime}$, then $\Gamma \vdash e_{1}^{\prime} \leq e_{2}^{\prime}: A$.

Now the proof by induction can proceed with the generalized lemma. For the case which was stuck in the previous attempt, the conclusion is exactly the induction hypothesis that follows the case (1) of the lemma. The non-trivial case is when the derivation ends with the subsumption rule S-SuB. When $e_{1}$ and $e_{2}$ are not both cast $\uparrow$ terms, the proof is trivial by the induction hypothesis. Otherwise, we have $e_{1}=\operatorname{cast}_{\uparrow}[C] e_{1}^{\prime}$ and $e_{2}=\operatorname{cast}_{\uparrow}[C] e_{2}^{\prime}$ such that

$$
\begin{array}{lll}
\Gamma \vdash \operatorname{cast}_{\uparrow}[C] e_{1}^{\prime} \leq \operatorname{cast}_{\uparrow}[C] e_{2}^{\prime}: B & \text { (1) } & \Gamma \vdash B \leq A: \star \\
A \hookrightarrow A^{\prime} & \text { (3) } & C \hookrightarrow C^{\prime}
\end{array}
$$

Our target is to show $\Gamma \vdash e_{1}^{\prime} \leq e_{2}^{\prime}: A^{\prime}$. Note that the annotations of cast $\uparrow$ in both terms must be the same (i.e. $C$ ) by S-CAstUp. By inversion of (1), we have $\Gamma \vdash C \leq B: \star$. We first show there exists some $B^{\prime}$ such that $B \hookrightarrow B^{\prime}$ by proving the following lemma:

Lemma 4.16 (Reduction Exists in the Middle). Given that $\Gamma \vdash C \leq B: D$ and $\Gamma \vdash B \leq A: D$, if $C \hookrightarrow C^{\prime}$ and $A \hookrightarrow A^{\prime}$, then there exists $B^{\prime}$ such that $B \hookrightarrow B^{\prime}$.

Then by induction hypothesis, we have $\Gamma \vdash e_{1}^{\prime} \leq e_{2}^{\prime}: B^{\prime}$ from (1) by the first case of lemma and $\Gamma \vdash B^{\prime} \leq A^{\prime}: \star$ from (2) by the second (1st case impossible). Thus, we can prove the target by S-SuB 
and conclude Lemma 4.15. Finally, it is trivial to show that the original subtype preservation lemma is a corollary of the generalized one. Thus, we can conclude the type preservation lemma which is an immediate corollary of subtype preservation.

Progress. Progress states that well-formed terms do not get stuck:

Lemma 4.17 (Progress). If $\varnothing \vdash e:$ A then either $e$ is a value $v$ or there exists $e^{\prime}$ such that $e \hookrightarrow e^{\prime}$.

As we mentioned in Section 3.2, the type-level reduction in cast operators may encounter open terms. We prove a stronger progress lemma with a non-empty context:

Lemma 4.18 (Generalized Progress). If $\Gamma \vdash e:$ A then either $e$ is a value $v$ or there exists $e^{\prime}$ such that $e \hookrightarrow e^{\prime}$.

Then the original progress lemma is an immediate corollary of the stronger version. The proof is straightforward by induction on the derivation of $\Gamma \vdash e: A$. The definition of values is critical to the proof as it covers many stuck terms with variables and the top type (see also the discussion of inert terms in Section 3.2).

\section{ALGORITHMIC VERSION}

As we mentioned in Section 3.3, the unified subtyping judgment presented in Figure 3 is declarative but almost algorithmic. The typing part is declarative because of the subsumption rule, while the subtyping part is algorithmic. If we separately check the typing part and subtyping part, we just need to develop an algorithm for type checking. We use bidirectional type checking [Pierce and Turner 2000], a standard technique to develop the type checking algorithm for type systems with subtyping. We only briefly introduce the bidirectional system without showing rules which are mostly standard. For space reasons, the full specification is omitted and can be found in the extended version [Yang and Oliveira 2017]. We show the soundness and completeness of the type and subtype checking algorithm with respect to the original unified subtyping judgment. The full proof (mechanized in Coq) is available in the extended version [Yang and Oliveira 2017]. Developing a unified algorithmic system is left as future work, as will be discussed in Section 7.

\subsection{Bidirectional Type Checking}

We extend the syntax of $\lambda I_{\leq}$with annotations, denoted by ( $\left.e: A\right)$ (parentheses are required). We use $|e|$ to denote the erasure of all annotation from a term and $|\Gamma|$ for the erasure of a context. The algorithmic subtyping judgment is denoted by $\Gamma \vdash e_{1} \leq e_{2}$. It is developed by removing the typing part of unified subtyping rules in Figure 3.

The algorithmic typing judgment has two directions: the checking judgment $\Gamma \vdash e \Leftarrow A$ and the synthesis judgment $\Gamma \vdash e \Rightarrow A$. They are developed by following the typing part of unified subtyping. Most syntactic forms are typed by the synthesis judgment, including functions and function types since both binders are annotated. Two syntactic forms that are not annotated require the checking judgment, namely the top type $(T)$ and cast $\downarrow_{\downarrow}$ term. The subsumption rule from the unified subtyping is adapted to the checking direction.

We use erasure in the typing judgment to ensure there are no annotations in 1) the typing result and the context, 2) the terms being compared by the algorithmic subtyping judgment, and 3) the terms checked by the reduction relation $(\hookrightarrow)$. However, if erasure is used in the typing result of a premise using synthesis, i.e., $\Gamma \vdash e \Rightarrow|A|$, the original form of $A$ requires guessing. Referring to the original $A$ in other premises renders the typing rule not algorithmic. Thus, we make sure there is no such form of synthesis in the premises of typing rules. Note that the typing rule is still algorithmic if the erased typing result appears in the conclusion of a synthesis rule or a premise using checking judgment. 


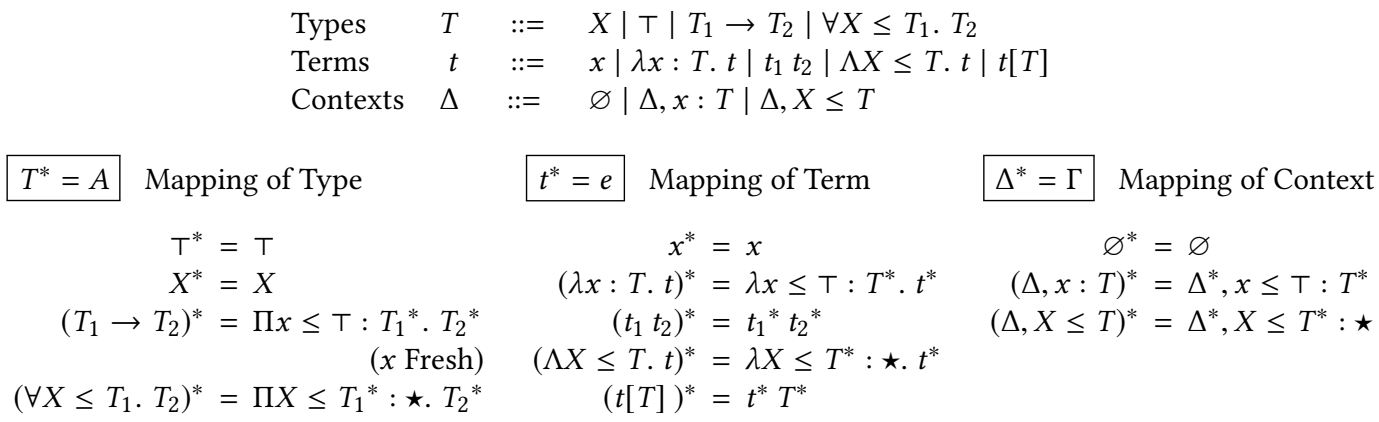

Fig. 5. Syntax and Translation of System $F_{\leq}$

\subsection{Soundness and Completeness}

We show that the algorithmic subtyping and typing are both sound and complete to the original unified subtyping. We use $\Gamma \vdash e \Leftrightarrow A$ to denote a judgment which can either be the checking judgment $\Gamma \vdash e \Leftarrow A$ or the synthesis judgment $\Gamma \vdash e \Rightarrow A$. The main theorems are stated as follows:

Theorem 5.1 (Soundness of Algorithm). If $\Gamma \vdash e_{1} \Leftrightarrow A, \Gamma \vdash e_{2} \Leftrightarrow A$ and $\Gamma \vdash e_{1} \leq e_{2}$, then $|\Gamma| \vdash\left|e_{1}\right| \leq\left|e_{2}\right|:|A|$.

Theorem 5.2 (Completeness of Algorithm). If $\Gamma \vdash e_{1} \leq e_{2}: A$, then $\Gamma \vdash e_{1} \leq e_{2}$ and there exists $e_{1}^{\prime}$ and $e_{2}^{\prime}$ such that $\Gamma \vdash e_{1}^{\prime} \Rightarrow A$ and $\Gamma \vdash e_{2}^{\prime} \Rightarrow A$ with $\left|e_{1}^{\prime}\right|=e_{1}$ and $\left|e_{2}^{\prime}\right|=e_{2}$.

\section{SUBSUMPTION OF SYSTEM $F_{\leq}$}

$\lambda I_{\leq}$is a generalization of System $F_{\leq}$with dependent types. In this section, we show that $\lambda I_{\leq}$can completely subsume the Kernel Fun variant [Cardelli and Wegner 1985] of System $F_{\leq}$. We first show the translation from System $F_{\leq}$to $\lambda I_{\leq}$and prove that the typing and subtyping judgments of System $F_{\leq}$still hold in $\lambda I_{\leq}$up to mapping. The full proofs and specification of System $F_{\leq}$are available in the extended version of this paper [Yang and Oliveira 2017].

\subsection{Translating System $F_{\leq}$to $\lambda I_{\leq}$}

We show the syntax of System $F_{\leq}$and mapping (denoted by ${ }^{*}$ ) of types, terms and contexts from System $F_{\leq}$to $\lambda I_{\leq}$in Figure 5. We use the metavariable $T$ for types, $t$ for terms and $\Delta$ for contexts in System $F_{\leq}$. The arrow type is non-dependent and unbounded and therefore mapped to a topbounded function type, similar to the treatment of syntactic sugar in Figure 1. The universal type is mapped to the dependent function type since $X$ can appear in $T_{2}$. The bound $T_{1}$ is a proper type and mapped to $T_{1}{ }^{*}$ with kind $\star$. The term and type abstraction, as well as term and type binding of the context, are treated similarly. Other mappings hold few surprises.

\subsection{Subsumption of Typing and Subtyping}

We prove that the mapped typing and subtyping relations still hold in $\lambda I_{\leq}$. The type system of System $F_{\leq}$we used here is the algorithmic [Curien and Ghelli 1992] and Kernel Fun variant [Cardelli and Wegner 1985]. We first show the well-formedness of types and contexts still hold after mapping:

LEMMA 6.1 (MAPPING OF WeLL-Formedness). (1) If $\Delta \vdash T$, then $\Delta^{*} \vdash T^{*}: \star$; (2) If $\vdash \Delta$, then $\vdash \Delta^{*}$

The proof is by simultaneous induction on the derivation of well-formedness of types $\Delta \vdash T$ and contexts $\vdash \Delta$. Then we show the mapped subtyping and typing still hold: 
Theorem 6.2 (Subsumption of Subtyping). If $\Delta \vdash T_{1} \leq T_{2}$, then $\Delta^{*} \vdash T_{1}{ }^{*} \leq T_{2}{ }^{*}: \star$.

Theorem 6.3 (Subsumption of Typing). If $\Delta \vdash t: T$, then $\Delta^{*} \vdash t^{*}: T^{*}$.

The proof is straightforward by induction on the derivation of subtyping relation $\Delta \vdash T_{1} \leq T_{2}$ and typing relation $\Delta \vdash t: T$, respectively. Note that the mapped typing relation $\Delta^{*} \vdash t^{*}: T^{*}$ is syntactic sugar of unified subtyping relation, i.e., $\Delta^{*} \vdash t^{*} \leq t^{*}: T^{*}$ (see Figure 1).

\section{DISCUSSION}

In this section, we discuss alternative designs for $\lambda I_{\leq}$and justify their trade-offs to the current design.

Recursion and Recursive Types. The current syntax of $\lambda I_{\leq}$does not contain any form of recursion. Adding recursion and recursive types is easy by simply following the treatment of recursion in $\lambda I$ [Yang et al. 2016]. We have an alternative formulation of our system (including full proofs) with those features. However subtyping recursive types reveals an interesting problem. The typical Amber rule [Cardelli 1986a], or even the following restricted invariant rule

$$
\frac{\Gamma, x \leq \mathrm{T}: A \vdash e_{1} \leq e_{2}: A \quad \Gamma \vdash A: \star}{\Gamma \vdash\left(\mu x: A . e_{1}\right) \leq\left(\mu x: A . e_{2}\right): A} \mathrm{~S}-\mathrm{MUI}
$$

does not work well with $\lambda I_{\leq}$. Here $\mu x: A$. $e_{1}$ is a recursive type with the recursive binder $x$ that can appear in the body $e_{1}$. The rule requires the types of recursive binders to be the same. We add a new reduction rule to unroll a recursive type: $\mu x: A . e \hookrightarrow e[x \mapsto \mu x: A$. $e]$. In order to keep type soundness, we need to ensure subtype preservation (Lemma 4.14) still holds. If $f=\lambda y: \star . y$ is an identity type operator with type $\star \rightarrow \star$, consider

$$
\mu x: \star . f x \leq \mu x: \star . \top x
$$

This relation holds by the rule S-MuI because we have $f \leq T: \star \rightarrow \star$ by S-Top and then $x: \star \vdash f x \leq \top x: \star$ by S-App. Subtype preservation requires that the subtyping relation still holds with both sides reduced by one step:

$$
f(\mu x: \star . f x) \leq \top(\mu x: \star . \top x)
$$

However, (1) does not hold because the pointwise subtyping rule S-App requires arguments of two applications should be the same. Thus, types are not preserved using the invariant rule for subtyping recursive types. This issue appears to be common to most systems with higher-order subtyping [Aspinall and Compagnoni 1996; Pierce and Steffen 1997; Zwanenburg 1999], as it arises from the interaction between the rules for recursive types and rules that use pointwise subtyping.

To solve this issue, we either change the S-App rule to be polarized [Steffen 1998], or only allow subtyping two identical recursive types. The former approach is interesting, but requires a major modification to the system. We leave that approach for future work. The latter approach is relatively simple by using the following rule:

$$
\frac{\Gamma, x \leq \mathrm{T}: A \vdash e: A \quad \Gamma \vdash A: \star}{\Gamma \vdash(\mu x: A . e) \leq(\mu x: A . e): A} \mathrm{~S}-\mathrm{MU}
$$

Due to the unified syntax, $\mu x: A$. $e$ can serve as both the term-level fixpoint and recursive type. Though full subtyping of recursive types is not possible in $\lambda I_{\leq}$currently, we are still able to introduce general recursion and recursive types to the system with S-Mu. This is precisely the approach used in our alternative formulation. 
Operational Semantics. $\lambda I_{\leq}$uses the same call-by-name (CBN) operational semantics that $\lambda I$ [Yang et al. 2016] uses. However most OO languages use call-by-value (CBV). CBV semantics is more complicated because of the existence of dependent types and explicit casts in $\lambda I_{\leq}$, but we believe that it should also be possible to have a variant of the calculus with CBV. We also treat cast $\uparrow[A] e$ as a value (see Section 3.2), which follows the standard call-by-name semantics of iso-recursive types [Harper 2013]. Such design makes the cast $\uparrow$ operator computationally relevant. Alternatively, we can take the approach from $\lambda I$, which treats cast $\uparrow_{\uparrow}[A] v$ as a value and adds a reduction rule to further reduce the inner term of cast $\uparrow$. However, the alternative semantics of cast $\uparrow$ leads to more complex reduction rules and metatheory. The cast canceling rule R-CASTELIm (See Figure 2) now needs to check if the inner term of cast $\uparrow$ is a value, which requires some non-trivial changes to current proofs of the metatheory. We leave the CBV semantics and computational irrelevance of casts as future work.

Top Types. For top types, we can alternatively treat only $T$ as a value but not $T e_{1} \ldots e_{n}$, which is an inert term (see Figure 1). In such design additional reduction rules similar to the $\beta$ T-reduction rules of System $F_{\leq}^{\omega}$ [Pierce and Steffen 1997] are needed to further reduce "stuck" terms to values, i.e., $\top e \hookrightarrow T$. However, the approach of using $\beta$ T-reduction needs to define reduction rules for each form of stuck terms, e.g., $T e$ and cast $\downarrow T$, while the definition of inert terms deals with stuck terms in a more uniform way.

Weak vs Full Casts. Cast operators in $\lambda I_{\leq}$use the same weak-head reduction for type-level computation. As mentioned in Section 2.4, certain type conversions cannot be performed by weakhead reduction/expansion if they require reduction at non-head position, e.g., converting $\operatorname{Vec}(1+1)$ to Vec 2. To address this limitation we can use an alternative design from the $\lambda I_{\mathrm{p}}$ variant [Yang et al. 2016] of $\lambda I$. In that design full reduction is used in cast operators, which allows reduction at any position of a term. However the metatheory of $\lambda I_{\mathrm{p}}$ variant is significantly more complicated than the weak-head version. Since weak-head reduction was simpler and sufficient for our purposes (to model object encodings) we opted for that variant. It would be interesting to study the full-cast variant of $\lambda I_{\mathrm{p}}$ with subtyping as well in future work.

Unified Algorithmic System. In Section 5, we present an algorithmic version of $\lambda I_{\leq}$. A notable difference from the declarative system is that the typing and subtyping relation are defined separately. An alternative design is to create an algorithmic unified subtyping relation directly from the declarative version. The checking and synthesis judgments are denoted by $\Gamma \vdash e_{1} \leq e_{2} \Leftarrow A$ and $\Gamma \vdash e_{1} \leq e_{2} \Rightarrow A$, respectively. However, the design is only a sketch and currently we do not have a completeness proof for the unified algorithmic system.

Full Contravariance of Function Types. As mentioned in Section 3.3, the unified subtyping rule of function types is partially contravariant in the sense that bounds of function types are identical, which follows the treatment of universal types in the Kernel Fun variant [Cardelli and Wegner 1985] of System $F_{\leq}$. An alternative is to follow the full System $F_{\leq}$that allows bounds to be contravariant:

$$
\begin{aligned}
& \Gamma \vdash A^{\prime} \leq A: \star \quad \Gamma \vdash e^{\prime} \leq e: A \quad \Gamma, x \leq e^{\prime}: A^{\prime} \vdash B \leq B^{\prime}: \star \\
& \Gamma \vdash e: A \quad \Gamma \vdash e^{\prime}: A^{\prime} \quad \Gamma \vdash A: \star \quad \Gamma, x \leq e: A \vdash B: \star \\
& \hline \text { S-ProdF }
\end{aligned}
$$

We formulated an alternative system with such full contravariant rule and proved all lemmas in Section 4 still hold. The corresponding Coq formalization can be found with the companion materials of this paper available online. However, full System $F_{\leq}$is proved to be undecidable [Pierce 1992]. With contravariance of bounds, $\lambda I_{\leq}$using rule S-ProdF can subsume full System $F_{\leq}$, rendering 
the system undecidable. Though we have not proved the decidability of $\lambda I_{\leq}$yet, we adopt the Kernel-Fun rule in $\lambda I_{\leq}$and can at least rule out the undecidability caused by the full contravariance.

\section{RELATED WORK}

Subtyping with Unified Syntax. It is appealing to combine subtyping with the unified syntax of Pure Type Systems [Barendregt 1991] (PTS) for obtaining a concise and expressive system. Chen proposed $\lambda C_{\leq}$[Chen 1997], an extension of the calculus of constructions $(\lambda C)$ with subtyping. $\lambda C_{\leq}$ supports neither top types nor bounded quantification in order to simplify the metatheory. The proof of transitivity in $\lambda C_{\leq}$is simpler and does not depend on strong normalization, though decidability still depends on strong normalization as in $\lambda C$. Zwanenburg proposed PTS $\leq$ [Zwanenburg 1999] by extending PTS with subtyping and bounded quantification. It has the PTS-style unified syntax but with two distinct forms of abstraction for type and bound. In $P T S^{\leq}$, the subtyping rules do not depend on the typing rules, which allows proving subtyping properties independently from typing properties. However, such design makes it difficult to extend the framework with two desirable features: 1) subtyping on bounded abstractions, since subtyping rules are defined only for pre-terms; 2) top types, since the subtyping rule of top types depends on typing. Neither of those features are supported by $P T S^{\leq}$.

Hutchins proposed another framework called Pure Subtype Systems [Hutchins 2010] (PSS) which also adopts the unified syntax based on PTS. The design is simplified by making the system solely based on subtyping without the typing relation. The simplicity of the system comes at the cost of the complexity of metatheory. The proof of transitivity elimination is partial, and therefore subject reduction cannot be proved. Note that although $\lambda I_{\leq}$shares the similar idea of being based on the subtyping relation, it has two major differences from PSS. First, $\lambda I_{\leq}$unifies subtyping with typing in a more conservative way. The unified subtyping relation still tracks types and it intuitively subsumes the traditional typing relation. In contrast, PSS takes a more aggressive approach to make the typing relation completely absent from the system. In PSS there are no types or typing. Second, PSS eliminates the distinction of function and function types, which are unified into the same syntax of abstraction. In contrast, $\lambda I_{\leq}$still distinguishes these two concepts as in PTSs. Since the subtyping rule of abstractions in PSS is pointwise, any form of contravariance is not supported. An unfortunate consequence is that PSS cannot subsume System $F_{\leq}$with contravariant arrow types, including the Kernel Fun variant [Cardelli and Wegner 1985].

Stratified Syntax with High-Order Subtyping. System $F_{\leq}^{\omega}$ is a lambda calculus with stratified syntax by extending System $F_{\omega}$ [Girard 1972] with higher-order subtyping. To simplify the metatheory, early formalizations of System $F_{\leq}^{\omega}$ [Compagnoni 1995; Pierce and Steffen 1997] do not allow a bounded type operator. Compagnoni and Goguen later proposed a technique called typed operational semantics [Compagnoni and Goguen 2003] to fully enable bounded quantification in System $F_{\leq}^{\omega}$. But its metatheory becomes quite complicated and relies on strong normalization, making it hard to apply such technique to systems with general recursion. Note that Compagnoni and Goguen's presentation of System $F_{\leq}^{\omega}$ contains a kinded subtyping judgment $\Gamma \vdash A \leq B: K$ which has a similar shape to the unified subtyping relation in $\lambda I_{\leq}$. But the typing relation is separately defined in their system and not subsumed by the kinded subtyping judgment.

Stratified Subtyping Systems with Dependent Types. System $\lambda P_{\leq}$[Aspinall and Compagnoni 1996] is a stratified system with dependent types and higher-order subtyping. The metatheory becomes more complex than System $F_{\leq}^{\omega}$ due to the circular dependency of typing, kinding and subtyping. A novel proof technique that splits beta reduction on terms and types is proposed to break such dependency. However, System $\lambda P_{\leq}$does not support polymorphism (i.e. abstraction over types), bounded quantification or top types. System $\lambda \Pi_{\leq}$[Castagna and Chen 2001; Chen 1998] is an 
improvement of $\lambda P_{\leq}$. It has the property of type-level transitivity elimination, while System $\lambda P_{\leq}$ has transitivity elimination only for normalized types. However, $\lambda \Pi_{\leq}$is proved to be equivalent to $\lambda P_{\leq}$in typing and subtyping, meaning that it has no increased expressiveness.

Subtyping with Restricted Dependent Types. There have been several studies focusing on exploring subtyping with restricted forms of dependent types but not full dependent types in the context of object-oriented (OO) programming. The Dependent Object-Oriented Language [Campos and Vasconcelos 2015] (DOL) is an imperative OO programming language with subtyping and index refinements, a restricted notion of dependent types originated from Dependent ML [Xi and Pfenning 1999], which allows types to depend on static indices of natural numbers. DOL supports the verification of mutable objects and unrestricted use of shared objects. The type checking of DOL is decidable. However, the metatheory of DOL is not fully developed yet.

$v O b j$ [Odersky et al. 2003] is a dependently typed calculus for objects with type members. It is developed as a theoretic foundation for Scala [Odersky et al. 2004] and features a weaker form of dependent types called path-dependent types. In $v O b j$, types can depend on paths which are type selections on variables, i.e., $x . L$. Compared to traditional dependent types used in $\lambda I_{\leq}$, it is difficult to use path-dependent types to model dependency on non-path values, e.g., $\Pi n:$ Int. Vec $n$. The richness of the type system makes the metatheory of $v O b j$ complex and type checking is not decidable. Another recent effort of developing a core calculus for Scala is the Dependent Object Types (DOT) calculus [Amin et al. 2016, 2012, 2014; Rompf and Amin 2016]. DOT is also based on path-dependent types. It is simpler and has fewer type forms than $v O b j$, e.g., no class types, but still expressive to model many features of Scala. Similarly to $\lambda I_{\leq}$, DOT subsumes System $F_{\leq}$but has a richer notion of bounds. Type variables can be quantified by both lower bounds and upper bounds, as opposed to the traditional bounded quantification used in $\lambda I_{\leq}$that only supports upper bounds. The metatheory of DOT is well-developed [Rompf and Amin 2016], though the soundness proof requires many non-standard techniques. Transitivity of subtyping needs to be treated as an axiom and transitivity elimination is not possible [Rompf and Amin 2016]. Both $v O b j$ and DOT use the stratified syntax in contrast to the unified syntax of $\lambda I_{\leq}$

Dependent Types with Explicit Casts. One key difference of $\lambda I_{\leq}$to other systems with higherorder subtyping is the absence of a conversion rule. Instead, explicit casts are used for performing type-level computation. The motivation of using casts in $\lambda I_{\leq}$is to decouple strong normalization from the proofs of metatheory, which also makes it possible to allow general recursion. There have been several studies [Kimmell et al. 2012; Sjöberg et al. 2012; Sjöberg and Weirich 2015; Stump et al 2008; van Doorn et al. 2013; Weirich et al. 2013; Yang et al. 2016] working on using explicit casts instead of conversion rule in a dependently typed system. We follow the iso-types approach from $\lambda I$ calculus [Yang et al. 2016] which is a generalization of iso-recursive types. However, $\lambda I$ and other mentioned studies do not deal with subtyping in their systems.

\section{CONCLUSION AND FUTURE WORK}

This paper presented the $\lambda I_{\leq}$calculus, a dependently typed calculus with unified syntax, which supports higher-order polymorphism, bounded quantification and top types. The calculus unifies typing and subtyping into a single relation, eliminating the circularity of typing and subtyping. The transitivity and type safety of $\lambda I_{\leq}$are proved. For the future, we intend to show the decidability of $\lambda I_{\leq}$. We already have a sound and complete algorithmic system and we believe it is decidable, though we do not have the proof yet. We also hope to explore several alternative designs of the calculus, such as full subtyping of recursive types, casts with full reduction and unified algorithmic subtyping. We would also like to incorporate the more general kinds of bounds of DOT into $\lambda I_{\leq}$, and study whether that would suffice for $\lambda I_{\leq}$to express common Scala programming idioms.

Proc. ACM Program. Lang., Vol. 1, No. OOPSLA, Article 47. Publication date: October 2017. 


\section{ACKNOWLEDGMENTS}

We thank the anonymous reviewers and Tom Schrijvers for their helpful comments. We use the Ott tool [Sewell et al. 2010] for typesetting and formalization of this paper. This work has been sponsored by the Hong Kong Research Grant Council projects number 27200514 and 17258816.

\section{REFERENCES}

Andreas Abel and Dulma Rodriguez. 2008. Syntactic metatheory of higher-order subtyping. In CSL '08. 446-460.

Beniamino Accattoli and Giulio Guerrieri. 2016. Open Call-by-Value. In APLAS '16. Springer, 206-226.

Thorsten Altenkirch, Nils Anders Danielsson, Andres Löh, and Nicolas Oury. 2010. $\Pi \Sigma$ : Dependent types without the sugar. In Functional and Logic Programming. Springer, 40-55.

Nada Amin, Samuel Grütter, Martin Odersky, Tiark Rompf, and Sandro Stucki. 2016. The essence of dependent object types. In A List of Successes That Can Change the World. Springer, 249-272.

Nada Amin, Adriaan Moors, and Martin Odersky. 2012. Dependent object types. In FOOL '12. ACM.

Nada Amin, Tiark Rompf, and Martin Odersky. 2014. Foundations of path-dependent types. In OOPSLA '14. ACM, 233-249.

David Aspinall and Adriana Compagnoni. 1996. Subtyping dependent types. In LICS '96. 86-97.

Lennart Augustsson. 1998. Cayenne - a Language with Dependent Types. In ICFP '98. ACM, 239-250.

Henk Barendregt. 1991. Introduction to generalized type systems. fournal of Functional Programming 1, 2 (1991), $125-154$.

Henk Barendregt. 1992. Lambda Calculi with Types. In Handbook of Logic in Computer Science, Vol. 2. 117-309.

Edwin Brady. 2013. Idris, a general-purpose dependently typed programming language: Design and implementation. Fournal of Functional Programming 23, 05 (2013), 552-593.

Kim B. Bruce, Luca Cardelli, and Benjamin C. Pierce. 1999. Comparing object encodings. Information and Computation 155, 1-2 (1999), 108-133.

Joana Campos and Vasco T. Vasconcelos. 2015. Imperative objects with dependent types. In FTffP '15. ACM, 2:1-2:6.

Luca Cardelli. 1986a. Amber. In Combinators and Functional Programming Languages. Springer Berlin Heidelberg, 21-47.

Luca Cardelli. 1986b. A Polymorphic lambda-calculus with Type: Type. Digital Systems Research Center.

Luca Cardelli, Simone Martini, John C. Mitchell, and Andre Scedrov. 1994. An extension of system F with subtyping. Information and Computation 109, 1-2 (1994), 4-56.

Luca Cardelli and Peter Wegner. 1985. On understanding types, data abstraction, and polymorphism. ACM Computing Surveys (CSUR) 17, 4 (1985), 471-523.

Chris Casinghino, Vilhelm Sjöberg, and Stephanie Weirich. 2014. Combining Proofs and Programs in a Dependently Typed Language. In POPL '14. ACM, 33-45.

Giuseppe Castagna and Gang Chen. 2001. Dependent types with subtyping and late-bound overloading. Information and Computation 168, 1 (2001), 1-67.

Gang Chen. 1997. Subtyping calculus of construction. Mathematical Foundations of Computer Science 1997 (1997), $189-198$.

Gang Chen. 1998. Dependent type system with subtyping (I) type level transitivity elimination. fournal of Computer Science and Technology 13, 6 (1998), 564-578.

Gang Chen. 2003. Coercive Subtyping for the Calculus of Constructions. In POPL '03. ACM, 150-159.

Adriana Compagnoni and Healfdene Goguen. 2003. Typed operational semantics for higher-order subtyping. Information and Computation 184, 2 (2003), 242-297.

Adriana Beatriz Compagnoni. 1995. Higher-order subtyping with intersection types. Ph.D. Dissertation. University of Nijmegen.

Thierry Coquand and Gérard Huet. 1988. The Calculus of Constructions. Information and Computation 76 (1988), 95-120.

Karl Crary, Robert Harper, and Sidd Puri. 1999. What is a recursive module?. In PLDI '99. ACM, 50-63.

Pierre-Louis Curien and Giorgio Ghelli. 1992. Coherence of subsumption, minimum typing and type-checking in F $\leq$. Mathematical structures in computer science 2, 01 (1992), 55-91.

Jean-Yves Girard. 1972. Interprétation fonctionelle et élimination des coupures de l'arithmétique d'ordre supérieur. Ph.D. Dissertation. Université Paris VII.

Robert Harper. 2013. Practical foundations for programming languages (1st ed.). Cambridge University Press.

DeLesley S. Hutchins. 2010. Pure Subtype Systems. In POPL '10. ACM, 287-298.

Jonas Kaiser, Tobias Tebbi, and Gert Smolka. 2017. Equivalence of System F and $\lambda 2$ in Coq Based on Context Morphism Lemmas. In CPP '17. ACM, 222-234.

Garrin Kimmell, Aaron Stump, Harley D. Eades III, Peng Fu, Tim Sheard, Stephanie Weirich, Chris Casinghino, Vilhelm Sjöberg, Nathan Collins, and Ki Yung Ahn. 2012. Equational Reasoning about Programs with General Recursion and Call-by-value Semantics. In PLPV'12. 15-26.

Adriaan Moors, Frank Piessens, and Martin Odersky. 2008. Generics of a Higher Kind. In OOPSLA '08. ACM, 423-438. 
Ulf Norell. 2007. Towards a practical programming language based on dependent type theory. Ph.D. Dissertation. Chalmers University of Technology.

Martin Odersky, Philippe Altherr, Vincent Cremet, Burak Emir, Sebastian Maneth, Stéphane Micheloud, Nikolay Mihaylov, Michel Schinz, Erik Stenman, and Matthias Zenger. 2004. An Overview of the Scala Programming Language. Technical Report IC/2004/64. EPFL Lausanne, Switzerland.

Martin Odersky, Vincent Cremet, Christine Röckl, and Matthias Zenger. 2003. A nominal theory of objects with dependent types. In ECOOP '03. Springer, 201-224.

Luca Paolini and Simona Ronchi Della Rocca. 1999. Call-by-value Solvability. RAIRO-Theoretical Informatics and Applications 33, 6 (1999), 507-534

Benjamin C. Pierce. 1992. Bounded quantification is undecidable. In POPL '92. ACM, 305-315.

Benjamin C. Pierce. 2002. Types and programming languages. MIT press.

Benjamin C. Pierce and Martin Steffen. 1997. Higher-order subtyping. Theoretical computer science 176, 1 (1997), $235-282$.

Benjamin C. Pierce and David N. Turner. 1994. Simple type-theoretic foundations for object-oriented programming. fournal of functional programming 4, 02 (1994), 207-247.

Benjamin C. Pierce and David N. Turner. 2000. Local type inference. ACM Transactions on Programming Languages and Systems (TOPLAS) 22, 1 (2000), 1-44.

Tiark Rompf and Nada Amin. 2016. Type soundness for dependent object types (DOT). In OOPSLA '16. ACM, 624-641.

David A. Schmidt. 1994. The Structure of Typed Programming Languages. MIT Press.

Peter Sewell, Francesco Zappa Nardelli, Scott Owens, Gilles Peskine, Thomas Ridge, Susmit Sarkar, et al. 2010. Ott: Effective tool support for the working semanticist. Fournal of functional programming 20, 1 (2010), 71-122.

Vilhelm Sjöberg, Chris Casinghino, Ki Yung Ahn, Nathan Collins, Harley D. Eades III, Peng Fu, Garrin Kimmell, Tim Sheard, Aaron Stump, and Stephanie Weirich. 2012. Irrelevance, Heterogenous Equality, and Call-by-value Dependent Type Systems. In MSFP '12. 112-162.

Vilhelm Sjöberg and Stephanie Weirich. 2015. Programming Up to Congruence. In POPL '15. ACM, 369-382.

Martin Steffen. 1998. Polarized Higher-Order Subtyping. Ph.D. Dissertation. Technische Fakultät, Friedrich-Alexander-Universität Erlangen-Nürnberg.

Aaron Stump, Morgan Deters, Adam Petcher, Todd Schiller, and Timothy Simpson. 2008. Verified Programming in Guru. In PLPV '09. 49-58.

The Coq development team. 2016. The Coq proof assistant reference manual. https://coq.inria.fr/refman/ Version 8.6.

Floris van Doorn, Herman Geuvers, and Freek Wiedijk. 2013. Explicit Convertibility Proofs in Pure Type Systems. In LFMTP '13. ACM, 25-36.

Stephanie Weirich, Justin Hsu, and Richard A. Eisenberg. 2013. System FC with Explicit Kind Equality. In ICFP '13. ACM, 275-286.

Andrew K. Wright and Matthias Felleisen. 1994. A syntactic approach to type soundness. Information and computation 115 , 1 (1994), 38-94.

Hongwei Xi and Frank Pfenning. 1999. Dependent types in practical programming. In POPL '99. ACM, $214-227$.

Yanpeng Yang, Xuan Bi, and Bruno C. d. S. Oliveira. 2016. Unified Syntax with Iso-types. In APLAS '16. Springer, 251-270.

Yanpeng Yang and Bruno C. d. S. Oliveira. 2017. Unifying Typing and Subtyping (Extended version). Available from https://bitbucket.org/ypyang/oopsla17. (2017).

Jan Zwanenburg. 1999. Pure type systems with subtyping. In TLCA '99. 381-396. 